\title{
AN IMPROVED BOUND ON THE PACKING DIMENSION OF FURSTENBERG SETS IN THE PLANE
}

\author{
TUOMAS ORPONEN
}

\begin{abstract}
Let $0 \leq s \leq 1$. A set $K \subset \mathbb{R}^{2}$ is a Furstenberg $s$-set, if for every unit vector $e \in S^{1}$, some line $L_{e}$ parallel to $e$ satisfies

$$
\operatorname{dim}_{H}\left[K \cap L_{e}\right] \geq s .
$$

The Furstenberg set problem, introduced by T. Wolff in 1999, asks for the best lower bound for the dimension of Furstenberg $s$-sets. Wolff proved that $\operatorname{dim}_{H} K \geq \max \{s+1 / 2,2 s\}$ and conjectured that $\operatorname{dim}_{\mathrm{H}} K \geq(1+3 s) / 2$. The only known improvement to Wolff's bound is due to Bourgain, who proved in 2003 that $\operatorname{dim}_{\mathrm{H}} K \geq 1+\epsilon$ for Furstenberg $1 / 2$-sets $K$, where $\epsilon>0$ is an absolute constant. In the present paper, I prove a similar $\epsilon$-improvement for all $1 / 2<s<1$, but only for packing dimension: $\operatorname{dim}_{\mathrm{p}} K \geq 2 s+\epsilon$ for all Furstenberg $s$-sets $K \subset \mathbb{R}^{2}$, where $\epsilon>0$ only depends on $s$.

The proof rests on a new incidence theorem for finite collections of planar points and tubes of width $\delta>0$. As another corollary of this theorem, I obtain a small improvement for Kaufman's estimate from 1968 on the dimension of exceptional sets of orthogonal projections. Namely, I prove that if $K \subset \mathbb{R}^{2}$ is a linearly measurable set with positive length, and $1 / 2<s<1$, then

$$
\operatorname{dim}_{\mathrm{H}}\left\{e \in S^{1}: \operatorname{dim}_{\mathrm{p}} \pi_{e}(K) \leq s\right\} \leq s-\epsilon
$$
\end{abstract}

for some $\epsilon>0$ depending only on $s$. Here $\pi_{e}$ is the orthogonal projection onto the line spanned by $e$.

\section{CONTENTS}

1. Introduction 2

1.1. Furstenberg sets 2

1.2. Projections 3

1.3. Outline of the paper 4

1.4. Some notation 6

1.5. Acknowledgements 6

2. Reductions to Minkowski dimension and compact sets 6

3. Proofs of Theorems 2.6 and $2.8 \quad 8$

3.1. Finding the scale $\delta>0 \quad 8$

$\begin{array}{ll}\text { 3.2. } & \text { Finding } \delta \text {-tubes } \\ \text { 3.3. } & 10\end{array}$

3.3. Concluding the proofs of Theorems 2.6 and $2.8 \quad 14$

4. An incidence bound for points and tubes 14

4.1. The main counter assumption 16

2010 Mathematics Subject Classification. 28A80 (Primary).

Key words and phrases. Furstenberg sets, projections, packing dimension.

T.O. is supported by the Academy of Finland through the grant Restricted families of projections and connections to Kakeya type problems, grant number 274512. 
4.2. Considerations at scale $\delta^{1 / 2} \quad 16$

4.3. Refining the families of $\delta^{1 / 2}$-tubes 18

4.4. Considerations at scale $\delta \quad 21$

4.5. Quasi-product sets, and concluding the proof of Theorem $4.3 \quad 24$

4.6. An incidence theorem for quasi-product sets 25

References 31

\section{INTRODUCTION}

This paper is concerned with two closely related topics in planar fractal geometry: the Furstenberg set problem, and exceptional sets of orthogonal projections.

1.1. Furstenberg sets. I start with the central definition:

Definition 1.1 (Furstenberg sets). Let $0 \leq s \leq 1$. A set $K \subset \mathbb{R}^{2}$ is a Furstenberg $s$-set, if there exists a set of unit vectors $S_{K} \subset S^{1}$ with positive length such that the following holds: for every $e \in S_{K}$, some line $L_{e}$ parallel to $e$ satisfies $\operatorname{dim}_{H}\left[K \cap L_{e}\right] \geq s$. Here $\operatorname{dim}_{\mathrm{H}}$ is Hausdorff dimension.

The terminology was introduced in 1999 by T. Wolff [22], who proved the following lower bound for the Hausdorff dimension of Furstenberg sets:

Theorem 1.2 (Wolff's bound). The Hausdorff dimension of compact Furstenberg $s$-sets is at least $\max \{1 / 2+s, 2 s\}$.

Wolff suspected that his bound is not sharp, and made the following conjecture:

Conjecture 1.3 (Wolff's conjecture). The Hausdorff dimension of compact Furstenberg $s$-sets is at least $(1+3 s) / 2$.

Remark 1.4. Where does the name "Furstenberg set" come from? In 1970, H. Furstenberg [8] proved the following theorem. Assume that $p, q \in \mathbb{N}$ are integers such that $\log p / \log q$ is irrational. Assume that $A_{p}, A_{q} \subset[0,1]$ are closed sets invariant under $x \mapsto p x(\bmod 1)$ and $x \mapsto q x(\bmod 1)$, respectively. Let $0 \leq s \leq 1$, and assume that some line $L \subset \mathbb{R}^{2}$ satisfies $\operatorname{dim}_{\mathrm{H}}\left[L \cap\left(A_{p} \times A_{q}\right)\right] \geq s$. Then $A_{p} \times A_{q}$ is a Furstenberg $s$-set.

Furstenberg was interested in the problem: how do lines intersect product sets of the form $A_{p} \times A_{q}$ ? He conjectured that

$$
\operatorname{dim}_{\mathrm{H}}\left[L \cap\left(A_{p} \times A_{q}\right)\right] \leq \max \left\{0, \operatorname{dim}_{\mathrm{H}}\left(A_{p} \times A_{q}\right)-1\right\}
$$

for every line $L \subset \mathbb{R}^{2}$. Keeping in mind Furstenberg's theorem cited above, the upper bound (1.5) would evidently follow, if only one could prove the lower bound $\operatorname{dim}_{\mathrm{H}} K \geq$ $s+1$ for all Furstenberg $s$-sets. However, the estimate $\operatorname{dim}_{H} K \geq s+1$ is too optimistic in general: as shown by Wolff [22], Conjecture 1.3 is the strongest possible for general Furstenberg sets. In other words, the sets in Definition 1.1 are too general to help solve Furstenberg's conjecture (1.5) for the special sets of the form $A_{p} \times A_{q}$.

Fortunately, the services of general Furstenberg sets are no longer required for this purpose: only this year, Furstenberg's conjecture (1.5) was spectacularly verified (independently) by P. Shmerkin [20] and M. Wu [23], with two very different techniques! 
For general sets, progress in Conjecture 1.3 has been quite modest. Around the year 2000, Katz and Tao [9] observed that improving Wolff's bound for Furstenberg $\frac{1}{2}$-sets is roughly equivalent to proving a " $\delta$-discretised" sum-product theorem in $\mathbb{R}$. The latter task was then accomplished in 2003 by Bourgain [1]. Hence, the combined efforts of Katz-Tao and Bourgain give the following improvement to Wolff's bound:

Theorem 1.6 (Bourgain, Katz-Tao). There is an absolute constant $\epsilon>0$ such that the Hausdorff dimension of compact Furstenberg $\frac{1}{2}$-sets is at least $1+\epsilon$.

Of course, the theorem also gives an improvement for Furstenberg $s$-sets with $s$ very close to $\frac{1}{2}$, but, to the best of my knowledge, Wolff's bound remains the world record for other values of $s \in(0,1)$. The main purpose of this paper is to prove a Bourgain-KatzTao type $\epsilon$-improvement to Wolff's bound for all values $\frac{1}{2}<s<1$. As a notable caveat, the method only works for packing dimension:

Theorem 1.7. For $\frac{1}{2}<s<1$, there exists a constant $\epsilon=\epsilon(s)>0$ such that every Furstenberg $s$-set $K$ has packing dimension $\operatorname{dim}_{\mathrm{p}} K \geq 2 s+\epsilon$.

The foremost reason, why the proof of Theorem 1.7 does not give information about Hausdorff dimension - or even lower Minkowski dimension - is that it relies on the counter assumption $\operatorname{dim}_{\mathrm{p}} K \approx 2 s$, which gives information about $K$ on two different scales, namely $\delta$ and $\delta^{1 / 2}$. Assuming $\operatorname{dim}_{\mathrm{H}} K \approx 2 s$ does not have similar consequences. For a reader familiar with Besicovitch sets, I mention that a similar issue seems to stand in the way of improving Wolff's bound $\frac{5}{2}$ for the Hausdorff dimension of Besicovitch sets in $\mathbb{R}^{3}$ : the improved lower bound $\frac{5}{2}+\epsilon$ from 2000 by Katz, Łaba and Tao [10] is only known for upper Minkowski dimension. (Addendum to a second version of the paper: in April 2017, Katz and Zahl [11] posted on arXiv a proof that the Hausdorff dimension of Besicovitch sets in $\mathbb{R}^{3}$ is at least $\frac{5}{2}+\epsilon$.)

Finally, I mention that several papers have been written around Wolff's conjecture 1.3 in the past few years. An article of Zhang [24] completely solves a discrete variant of the conjecture, plus its analogues in higher dimensions. Zhang also studied a variant of the problem in finite fields [25]. Most recently, Ellenberg and Erman [3] used machinery from algebraic geometry to study a "k-plane" variant of Wolff's conjecture in finite fields.

1.2. Projections. The second topic of the paper are orthogonal projections. This is one of the most classical - and popular - topics in fractal geometry, so the amount of literature is immense: for a reader interested in finding out (much) more than covered below, I suggest taking a look at the recent survey of Fraser, Falconer and Jin [5].

Fix $0 \leq s \leq 1$, and let $K \subset \mathbb{R}^{2}$ be a Borel set of Hausdorff dimension $\operatorname{dim}_{\mathrm{H}} K \geq s$. In 1968, Kaufman [12] proved, improving an earlier result of Marstrand [14] from 1954, that

$$
\operatorname{dim}_{\mathrm{H}}\left\{e \in S^{1}: \operatorname{dim}_{\mathrm{H}} \pi_{e}(K)<s\right\} \leq s .
$$

Here $\pi_{e}: \mathbb{R}^{2} \rightarrow \mathbb{R}$ is the orthogonal projection $\pi_{e}(x)=x \cdot e$. Under the assumption $\operatorname{dim}_{H} K \geq s$, Kaufman's bound (1.8) is sharp: in 1975, Kaufman and Mattila [13] constructed explicit compact sets $K \subset \mathbb{R}^{2}$ with $\operatorname{dim}_{\mathrm{H}} K=s$ such that

$$
\operatorname{dim}_{\mathrm{H}}\left\{e: \operatorname{dim}_{\mathrm{H}} \pi_{e}(K)<s\right\}=s .
$$

Under the assumption $\operatorname{dim}_{\mathrm{H}} K \geq t>s$, the sharpness of (1.8) is an open problem. The following improvement is conjectured (in (1.8) of [15], for instance): 
Conjecture 1.10. Assume that $0 \leq t / 2 \leq s \leq t \leq 1$ and $\operatorname{dim}_{\mathrm{H}} K \geq t$. Then

$$
\operatorname{dim}_{\mathrm{H}}\left\{e \in S^{1}: \operatorname{dim}_{\mathrm{H}} \pi_{e}(K)<s\right\} \leq 2 s-t .
$$

It is well-known that there is a connection between the case $t=1$ of Conjecture 1.10 and Wolff's conjecture 1.3 for Furstenberg sets. As observed in 2012 by D. Oberlin [16], an improvement to Conjecture 1.10 immediately gives an improvement to Wolff's bound for Furstenberg sets arising from a special - but rather natural - construction. As far as I know, there is no published evidence of a converse, but it seems very likely that progress in Wolff's conjecture 1.3 would also lead to progress in Conjecture 1.10.

I now concentrate on the case $t=1$. If $0 \leq s \leq 1$ and $K \subset \mathbb{R}^{2}$ is a Borel set with $\operatorname{dim}_{\mathrm{H}} K \geq 1$, then $\operatorname{dim}_{\mathrm{H}} K \geq s$, and (1.8) holds for $K$. Curiously, it appears to be very difficult to capitalise on the stronger assumption $\operatorname{dim}_{\mathrm{H}} K \geq 1$, and beat the estimate (1.8). In fact, the only known improvement to Kaufman's bound (1.8) follows - once again - from Bourgain's discretised sum-product theorem. The next theorem appeared in another paper of Bourgain [2] from 2010:

Theorem 1.12 (Bourgain). Given $\kappa>0$, there exists $\eta>\frac{1}{2}$ such that the following holds. If $K \subset \mathbb{R}^{2}$ is a Borel set with $\operatorname{dim}_{\mathrm{H}} K \geq 1$, then $\operatorname{dim}_{\mathrm{H}} \pi_{e}(K) \geq \eta$ for all $e \in S^{1} \backslash E$, where $E \subset S^{1}$ is an exceptional set of Hausdorff dimension $\operatorname{dim}_{\mathrm{H}} E \leq \kappa$. In particular,

$$
\operatorname{dim}_{\mathrm{H}}\left\{e \in S^{1}: \operatorname{dim}_{\mathrm{H}} \pi_{e}(K) \leq s\right\} \rightarrow 0, \quad \text { as } s \searrow \frac{1}{2} .
$$

In brief, Theorem 1.12 marks a substantial improvement over Kaufman's bound (1.8) for values of $s$ very close to $\frac{1}{2}$, but for other values of $s \in(1 / 2,1)$, Kaufman's bound remains the world record. It is no coincidence that the situation is reminiscent of the known bounds for Furstenberg $s$-sets, for $s$ close to, or far from, $\frac{1}{2}$.

The second main result of the paper is a small improvement for the packing dimension variant of Kaufman's bound (1.8), for any $\frac{1}{2}<s<1$ :

Theorem 1.13. Let $\frac{1}{2}<s<1$. If $K \subset \mathbb{R}^{2}$ is an $\mathcal{H}^{1}$-measurable set with $\mathcal{H}^{1}(K)>0$, then

$$
\operatorname{dim}_{\mathrm{H}}\left\{e \in S^{1}: \operatorname{dim}_{\mathrm{p}} \pi_{e}(K) \leq s\right\} \leq s-\epsilon
$$

for some $\epsilon>0$ depending only on $\mathrm{s}$.

The reason for the appearance of $\operatorname{dim}_{\mathrm{p}}$ is the same as in Theorem 1.7, and the proof does not to give any improvement for the dimension of $\left\{e: \operatorname{dim}_{\mathrm{H}} \pi_{e}(K) \leq s\right\}$. The assumption $\mathcal{H}^{1}(K)>0$ is quite convenient, but nothing more: the proof would also work for Borel sets $K$ with $\operatorname{dim}_{\mathrm{H}} K \geq 1$.

Theorem 1.13 first appeared in a preliminary version of this paper [19] (which is now superseded by the current article, and hence not intended for publication). In the present paper, the proofs of Theorems 1.7 and 1.13 are deduced from a single discrete result, Theorem 3.12 below, which concerns incidences between certain finite families of points and $\delta$-tubes in the plane. At the level of this incidence result, Theorem 1.13 is strictly easier than Theorem 1.7, as the relevant families of $\delta$-tubes are somewhat special.

1.3. Outline of the paper. Both main results, Theorem 1.7 and 1.13 , will be proven simultaneously. Section 2 reduces the proofs to compact sets, and to corresponding claims about upper Minkowski dimension (instead of packing dimension). Section 3 reduces the proofs further to the discrete result mentioned above, namely Theorem 3.12. Section 4 - which is the main section of the paper - contains the proof of Theorem 3.12. 
The reductions to Theorem 3.12 are fairly standard, so Theorem 3.12 can be considered the main result of the paper. It states, roughly, the following: if every point $p$ in a $\delta$ discretised 1-dimensional set $P \subset \mathbb{R}^{2}$ is incident to a $\delta$-discretised $s$-dimensional set $\mathcal{T}_{p}$ of $\delta$-tubes, then at least one of the following holds. Either $\mathcal{T}=\cup_{p} \mathcal{T}_{p}$ contains $\gg \delta^{-2 s}$ tubes in total, or then it takes $\gg \delta^{-s}$ tubes of width $\delta^{1 / 2}$ to cover the union of the tubes in $\mathcal{T}$.

The proof has two phases: the first - and longer - reduces the proof to sets $P$ of a special form, which I call "quasi-product sets" in lack of a better term. This phase is elementary but tedious. One starts with a counter assumption: the union of the tubes in $\mathcal{T}$ can be covered by $\lesssim \delta^{-2 s}$ and $\lesssim \delta^{-s}$ tubes at scales $\delta$ and $\delta^{1 / 2}$, respectively. Building on this information, one eventually finds a single $\delta^{1 / 2}$ tube $T_{0}$ with the following properties. First, $T_{0}$ contains a large number of points from $P$. Second, each point in $P \cap T_{0}$ is incident to a large number of $\delta$-tubes $T$, which are essentially contained in $T_{0}$ (in particular, this can be used to find an upper bound on the total number of relevant tubes $T$ ). After such a $\delta^{1 / 2}$-tube $T_{0}$ has been found, one applies an affine re-scaling $A$, which essentially sends $P \cap T_{0}$ inside the unit square, and maps the $\delta$-tubes $T$ to $\delta^{1 / 2}$-tubes, see Figure 1 . Then, it turns out that $A\left(P \cap T_{0}\right)$ behaves like a quasi-product set, and has suspiciously many incidences with the $\delta^{1 / 2}$-tubes $A(T)$.

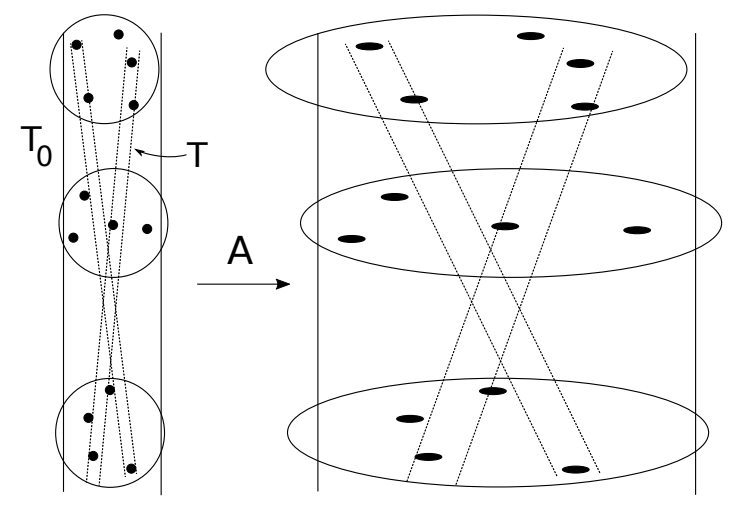

FIGURE 1 . The tubes $T_{0}, T$ and the set $P \cap T_{0}$, before and after the affine transformation $A$. Explaining why $A\left(P \cap T_{0}\right)$ "behaves like a quasiproduct set" would get too technical here, so I refer to Section 4.5 for more details.

At this point, it may seem like all the work has been fruitless: apart from changing scales from $\delta$ to $\delta^{1 / 2}$, the original incidence problem associated to $P$ and $\mathcal{T}$ has precisely the same numerology as the new incidence problem associated to $A\left(P \cap T_{0}\right)$ and the $\delta^{1 / 2}$ tubes $A(T)$. However, it turns out that the problem is easier to solve (or at least make progress in) for quasi-product sets, because tools from additive combinatorics become available.

In the second phase, one proves an incidence theorem for quasi-product sets (see Proposition 4.36). This uses standard tools from additive combinatorics, such as the Plünnecke-Ruzsa inequalities and the Balog-Szemerédi-Gowers theorem. In the end, it turns out that the incidence problem for quasi-product sets is roughly equivalent to a discretised variant of Bourgain's projection theorem, Theorem 1.12. Fortunately, Bourgain 
states and proves a suitable discretised variant of Theorem 1.12 in his paper [2], so the proof of Theorem 3.12 is completed by appealing to Theorem 5 in [2].

1.4. Some notation. An open ball in $\mathbb{R}^{d}$ with centre $x$ and radius $r>0$ will be denoted by $B(x, r)$. The Hausdorff measure and content of dimension $t$ will be denoted by $\mathcal{H}^{t}$ and $\mathcal{H}_{\infty}^{t}$, respectively. Given real numbers $A, B>0$, the notation $A \lesssim B$ means that $A \leq C B$ for some constant $C \geq 1$. If the dependence of $C$ on a parameter $p$ needs to be emphasised, I will write $A \lesssim p$. The notation $A \gtrsim B$ means that $B \lesssim A$, and $A \sim B$ stands for $A \lesssim B \lesssim A$.

The notation $A \lesssim \log B$ means that $A \lesssim \log ^{C}(1 / \delta) B$ for some absolute constant $C \geq 1$, where $\delta>0$ is a "scale". The meaning of $\delta>0$ will be clear from the context, whenever the notation is used. The notations $A \gtrsim \log B$ and $A \sim_{\log } B$ are then defined as above.

Given a bounded set $F \subset \mathbb{R}^{d}$, the notation $N(F, \delta)$ stands for the least number of balls of radius $\delta$ required to cover $F$. The upper Minkowski dimension of $F$ is

$$
\overline{\operatorname{dim}} F:=\limsup _{\delta \rightarrow 0} \frac{\log N(F, \delta)}{-\log \delta} .
$$

The packing dimension $\operatorname{dim}_{\mathrm{p}}$ is defined in (2.1) below.

1.5. Acknowledgements. I wish to thank the referees for reading the manuscript carefully and giving excellent comments; they helped me make the paper more readable.

\section{REDUCTIONS TO MINKOWSKI DIMENSION AND COMPACT SETS}

In this short section, I reduce the proofs of Theorems 1.7 and 1.13 to establishing analogous statements for Minkowski dimension (instead of packing dimension), and just for compact sets.

I start with reductions concerning Furstenberg sets. Let $K \subset \mathbb{R}^{2}$ be an arbitrary Furstenberg $s$-set, and let $S_{K} \subset S^{1}$ be the associated set of unit vectors with $\mathcal{H}^{1}\left(S_{K}\right)>0$. The definition of packing dimension is

$$
\operatorname{dim}_{\mathrm{p}} K=\inf \left\{\sup _{i} \overline{\operatorname{dim}} F_{i}: K \subset \bigcup_{i} F_{i}\right\},
$$

where the inf is taken over all countable covers of $K$ with bounded sets $F_{i}$. Since taking closures does not affect the upper Minkowski dimension $\overline{\mathrm{dim}}$, one may restrict attention to covers by compact sets $F_{i}$. Now, given any $\epsilon>0$, I claim that one of the sets $F_{i}$ is (essentially) a Furstenberg $(s-\epsilon)$-set. This is rather straightforward: since the sets $F_{i}$ cover $K$, one has

$$
\operatorname{dim}_{\mathrm{H}}\left[K \cap L_{e}\right]=\sup _{i} \operatorname{dim}_{\mathrm{H}}\left[F_{i} \cap L_{e}\right], \quad e \in S_{K},
$$

so for any fixed $e \in S^{1}$, it holds that $\mathcal{H}_{\infty}^{s-\epsilon}\left(F_{i} \cap L_{e}\right)>0$ for some $i$. Consequently, there exists $i$ such that $\mathcal{H}_{\infty}^{s-\epsilon}\left(F_{i} \cap L_{e}\right) \geq c>0$ for some $c>0$ and for a positive set of vectors $e$. Thus, $F_{i}$ is a Furstenberg $(s-\epsilon)$-set. Since $\epsilon>0$ was arbitrary, it follows that it suffices to prove Theorem 1.7 for the Minkowski dimension $\overline{\operatorname{dim}}$, for compact sets $K$, and under the extra assumption that

$$
\mathcal{H}_{\infty}^{s}\left(K \cap L_{e}\right) \geq c>0, \quad e \in S_{K}
$$


As a slightly less obvious reduction, I claim that, without loss of generality, one may assume that the lines $L_{e}, e \in S_{K}$, form a compact set. To formalise the statement, I recall the (standard) concept of point-line duality in the plane:

Definition 2.3 (Point-line duality). The points in $\mathbb{R}^{2}$ are in one-to-one correspondence with non-vertical lines in $\mathbb{R}^{2}$ via the mapping

$$
\mathcal{D}:(a, b) \mapsto\{y=a x+b: x \in \mathbb{R}\} .
$$

For every set of points $P$, define the set of lines $\mathcal{L}_{P}:=\mathcal{D}(P)=\{\mathcal{D}(p): p \in P\}$. Similarly, for a set $\mathcal{L}$ of non-vertical lines $\mathcal{L}$, define the set of points $P_{\mathcal{L}}:=\mathcal{D}^{-1}(\mathcal{L})$. A family of lines will be called open/closed/compact etc. if the point set $P_{\mathcal{L}}$ has the same topological property. I will also write $\mathcal{H}^{t}(\mathcal{L}):=\mathcal{H}^{t}\left(P_{\mathcal{L}}\right)$.

Remark 2.4. Even though the family of lines $\mathcal{D}(P)$ and the planar set $\cup\{L: L \in \mathcal{D}(P)\}$ are different objects, I will not differentiate between them in subsequent notation. In particular, if $B \subset \mathbb{R}^{2}$ is any set, the notation $B \cap \mathcal{D}(P)$ refers to $\cup\{B \cap L: L \in \mathcal{D}(P)\}$.

Now, let $\mathcal{L}_{K}:=\left\{L_{e}: e \in S_{K}\right\}$. Deleting a set of lines with sufficiently small measure, one may assume that every line in $\mathcal{L}_{K}$ makes a positive (and uniformly bounded from below) angle with the $y$-axis. Then $P_{K}:=P_{\mathcal{L}_{K}}$ is a bounded graph, that is, a set of the form $\{(a, f(a)): a \in A\}$, where $A \subset \mathbb{R}$ is a bounded set of positive length, and $f$ is a bounded function. Consider the compact line set

$$
\overline{\mathcal{L}}_{K}:=\mathcal{L}_{\bar{P}_{K}} .
$$

Then every line $L \in \overline{\mathcal{L}}_{K}$ has the property (2.2). This follows easily from the upper semicontinuity of Hausdorff content with respect to Hausdorff convergence and the compactness of $K$. Namely, assume for a moment that $L \in \overline{\mathcal{L}}_{K}$ is such that $K \cap L_{e}$ can be covered by finitely many open balls $B_{i}$ with

$$
\sum_{i} d\left(B_{i}\right)^{s}<c
$$

Choose a sequence of lines $L_{j} \in \mathcal{L}_{K}$ converging to $L$ locally in the Hausdorff metric. Then, using the compactness of $K$, the sets $K \cap L_{j}$ can also be covered by the balls $B_{i}$ for all $j$ large enough, contradicting (2.2).

The set $\bar{P}_{K}$ may no longer be a graph, but it certainly satisfies $\mathcal{H}^{1}\left(\bar{P}_{K}\right)>0$.

Definition 2.5 (Generalised Furstenberg $s$-set). Let $0<s<1$. Assume that $K \subset$ $B(0,1) \subset \mathbb{R}^{2}$ is a compact set, and $\mathcal{L}$ is a compact set of lines with $\mathcal{H}^{1}(\mathcal{L})>0$ with the property that $\mathcal{H}_{\infty}^{s}(K \cap L) \geq c>0$ for every $L \in \mathcal{L}$, and for some constant $c>0$. Also, assume that every line $L \in \mathcal{L}$ makes an angle $\geq 1 / 10$ with the $y$-axis. Then $K$ is called a generalised Furstenberg s-set.

By the previous discussion (and a simple coordinate-change, if necessary, to accommodate the angle requirement), the proof of Theorem 1.7 is now reduced to proving to following statement:

Theorem 2.6. $\overline{\operatorname{dim}} K \geq 2 s+\epsilon$ for every generalised Furstenberg $s$-set $K$, where $\epsilon>0$ only depends on $s$.

I now turn to the - much shorter - reduction related to the projection result, Theorem 1.13. The following observation is a special case Lemma 4.5 in [17]: 
Lemma 2.7. Assume that $K \subset \mathbb{R}^{2}$ is $\mathcal{H}^{1}$-measurable with $\mathcal{H}^{1}(K)>0$, and

$$
\operatorname{dim}_{\mathrm{H}}\left\{e \in S^{1}: \operatorname{dim}_{\mathrm{p}} \pi_{e}(K)<\sigma\right\}>\beta
$$

for some $\sigma, \beta>0$. Then, there exists a compact set $K^{\prime} \subset K$ with $\mathcal{H}^{1}\left(K^{\prime}\right)>0$ such that $\mathcal{H}^{1}\left(K^{\prime}\right)>0$ and

$$
\operatorname{dim}_{\mathrm{H}}\left\{e \in S^{1}: \overline{\operatorname{dim}} \pi_{e}\left(K^{\prime}\right)<\sigma\right\}>\beta .
$$

It follows immediately that it suffices to prove Theorem 1.13 for $\overline{\operatorname{dim}}$ instead of $\operatorname{dim}_{\mathrm{p}}$, and for compact sets $K$ with $\mathcal{H}^{1}(K)>0$. Also, one may restrict attention to the case $0<\mathcal{H}^{1}(K)<\infty$, since compact subsets with finite measure can always be found, and proving the theorem for any subset implies it for the whole set. For purposes of easy reference, I record the result explicitly:

Theorem 2.8. Let $1 / 2<s<1$. If $K \subset \mathbb{R}^{2}$ is a is compact with $0<\mathcal{H}^{1}(K)<\infty$, then

$$
\operatorname{dim}_{\mathrm{H}}\left\{e \in S^{1}: \overline{\operatorname{dim}} \pi_{e}(K) \leq s\right\} \leq s-\epsilon
$$

for some $\epsilon>0$ depending only on $s$.

\section{ProOfS OF THEOREMS 2.6 AND 2.8}

The proofs of both the main theorems are based on counter assumptions. If Theorem 2.6 fails, then for arbitrarily small $\epsilon>0$, there exists a generalised Furstenberg $s$-set $K_{F} \subset \mathbb{R}^{2}$ such that

$$
N\left(K_{F}, \delta\right) \leq \delta^{-2 s-\epsilon}
$$

for all small enough $\delta>0$, say $0<\delta \leq \delta_{1}$. Recall that $N(K, \delta)$ is the least number of balls of radius $\delta$ required to cover $K$. Similarly, if Theorem 2.8 fails, then for arbitrarily small $\epsilon>0$, there exists a number $1 / 2<s<1$, a compact set $K_{\pi} \subset \mathbb{R}^{2}$ with $0<\mathcal{H}^{1}\left(K_{\pi}\right)<\infty$, and a set of vectors $E \subset S^{1}$ with $\mathcal{H}^{s}(E)>0$ such that

$$
N\left(\pi_{e}\left(K_{\pi}\right), \delta\right) \leq \delta^{-s-\epsilon}, \quad e \in E,
$$

for all $0<\delta \leq \delta_{2}$.

The purpose of this section is to first pick the scale $0<\delta \leq \delta_{0} \leq \min \left\{\delta_{1}, \delta_{2}\right\}$ so that the information from the counter assumptions (3.1) and (3.2) is as strong as possible. Then, at this scale $\delta>0$, the counter assumptions are employed to construct an "impossible" configuration of $\delta$-separated points and $\delta$-tubes. The "impossibility" of the configuration is finally deduced from an incidence result, Theorem 3.12. The proof of the incidence result is a separate story, which will occupy the remainder of the paper.

3.1. Finding the scale $\delta>0$. The scale $\delta$ will only be chosen once, but it can be chosen arbitrarily small (by choosing $\delta_{0} \leq \min \left\{\delta_{1}, \delta_{2}\right\}$ very small). This will be useful - and implicitly assumed - countless times below. For example, I will always implicitly assume that $\delta^{-\epsilon}$ is far larger than various constants $C \geq 1$, which appear throughout the proof.

I start by recalling some basic facts about "discretising $s$-dimensional sets". As far as I know, the following definition is due to Katz and Tao [9]:

Definition $3.3\left((\delta, s, C)\right.$-sets). Fix $\delta, s>0$. A finite $\delta$-separated set $P \subset \mathbb{R}^{d}$ is called a $(\delta, s, C)$-set, if

$$
|P \cap B(x, r)| \leq C\left(\frac{r}{\delta}\right)^{s}
$$

for all $x \in \mathbb{R}^{d}$ and $\delta \leq r \leq 1$. Here and below, $|\cdot|$ stands for cardinality. 
An open ball of radius $\delta>0$ will be called a $\delta$-ball. A collection of $\delta$-balls will be called a $(\delta, s, C)$-set, if the centres of the balls form a $(\delta, s, C)$-set. The following proposition explains the rationale behind $(\delta, s, C)$-sets:

Proposition 3.5. Let $\delta>0$, and let $B \subset B(0,1) \subset \mathbb{R}^{2}$ be a set with $\mathcal{H}_{\infty}^{s}(B)=: \kappa>0$. Then, there exists $a(\delta, s, C)$-set $P \subset B$ with cardinality $|P| \geq(\kappa / C) \cdot \delta^{-s}$, where $C \geq 1$ is an absolute constant.

The proof is very close to that Frostman's lemma; the details can be found in the appendix of [7]. Note that any $(\delta, s, C)$-set $P \subset B(0,1)$ satisfies $|P| \leq C \delta^{-s}$. So, slightly informally, Proposition 3.3 says that sets $B \subset B(0,1)$ with $\mathcal{H}^{s}(B)>0$ contain $(\delta, s, C)$ sets with near-maximal cardinality.

Now, I will pick a suitable scale $\delta>0$. Recall the sets $K_{F}$ and $K_{\pi}$. Since $K_{F}$ is a generalised Furstenberg $s$-set, it comes bundled with a compact set of lines $\mathcal{L}$ with $\mathcal{H}^{1}(\mathcal{L})>0$. Clearly, one may also assume that $\mathcal{H}^{1}(\mathcal{L})<\infty$, since a compact finite-measure subset $\mathcal{L}^{\prime} \subset \mathcal{L}$ can be found, and then the pair $K_{F}, \mathcal{L}^{\prime}$ satisfies the same hypotheses as $K_{F}, \mathcal{L}$.

Let $K$ be either one of the sets $K_{\pi}$ or $P_{\mathcal{L}}$, so that $K \subset B(0,1)$ and $0<\mathcal{H}^{1}(K)<\infty$. I will treat $\mathcal{H}^{1}(K)$ as an absolute constant in the $\lesssim$-notation below; in particular $\mathcal{H}^{1}(K) \sim 1$. Let $\mu$ be a Frostman measure supported on $K$, that is, $\mu(K)=1$ and $\mu(B(x, r)) \lesssim r$ for all balls $B(x, r) \subset \mathbb{R}^{2}$. Next, let $\mathcal{B}$ be an efficient $\delta_{0}$-cover for $K$, that is,

$$
\sup \{\operatorname{diam} B: B \in \mathcal{B}\} \leq \delta_{0} \quad \text { and } \quad \sum_{B \in \mathcal{B}} \operatorname{diam}(B) \lesssim \mathcal{H}^{1}(K) \sim 1 .
$$

One may assume that the diameters of the balls in $\mathcal{B}$ are of the form $2^{-j}, j \in \mathbb{N}$. For $2^{-j} \leq \delta_{0}$, set $\mathcal{B}_{j}:=\left\{B \in \mathcal{B}: \operatorname{diam}(B)=2^{-j}\right\}$, and observe that

$$
\sum_{2^{-j} \leq \delta_{0}} \sum_{B \in \mathcal{B}_{j}} \mu(B) \geq \mu(K)=1 .
$$

In particular, there exists an index $j \in \mathbb{N}$ with $2^{-j} \leq \delta_{0}$ and

$$
\sum_{B \in \mathcal{B}_{j}} \mu(B) \gtrsim \frac{1}{\left(j-j_{0}+1\right)^{2}}
$$

Here $j_{0} \in \mathbb{N}$ is the smallest number with $2^{-j_{0}} \leq \delta_{0}$. Now, I set

$$
\delta:=2^{-2 j},
$$

so that $\delta^{1 / 2}=2^{-j}$. Note that $\delta^{1 / 2} \leq \delta_{0}$. In particular, (3.7) implies that

$$
\sum_{B \in \mathcal{B}_{j}} \mu(B) \gtrsim \log 1 \text {. }
$$

Observe that $\left|\mathcal{B}_{j}\right| \lesssim \delta^{-1 / 2}$ by (3.6), and on the other hand every ball $B \in \mathcal{B}_{j}$ satisfies $\mu(B) \lesssim \delta^{1 / 2}$. Thus, (3.8) implies that there are $\sim_{\log } \delta^{-1 / 2}$ balls in $\mathcal{B}_{j}$, denoted by $\mathcal{B}_{j}^{G}$, such that

$$
\mu(B) \gtrsim \log \delta^{1 / 2}, \quad B \in \mathcal{B}_{j}^{G} .
$$

Discarding a few balls if necessary, one may assume that

$$
\operatorname{dist}\left(B, B^{\prime}\right) \geq \delta^{-1 / 2}, \quad B, B^{\prime} \in \mathcal{B}_{j}^{G} .
$$


For each ball $B \in B_{j}^{G}$, choose a $(\delta, 1, C)$-set $P_{B} \subset B$ with $C \sim 1$ and $\left|P_{B}\right| \gtrsim \log \delta^{-1 / 2}$. This is possible by Proposition 3.5, since (3.9) and the linear growth of $\mu$ imply that $\mathcal{H}_{\infty}^{1}(B \cap$ $K) \gtrsim \log \delta^{1 / 2}$. Write

$$
P:=\bigcup_{B \in \mathcal{B}_{j}^{G}} P_{B}
$$

Then $|P| \sim_{\log } \delta^{-1}$, and $P_{B}=B \cap P$ for $B \in \mathcal{B}_{j}^{G}$. I will now verify that $P$ is a $(\delta, 1, C)$-set for some $C \sim_{\log } 1$. Fix $x \in P$ and $r \geq \delta$, let $B \in \mathcal{B}_{j}^{G}$ be the unique ball with $x \in P_{B}$. There are two cases to consider: if $\delta \leq r \leq \delta^{1 / 2}$, one needs only note that $|P \cap B(x, r)|=$ $\left|P_{B} \cap B(x, r)\right|$ by (3.10), and recall that $P_{B}$ is a $(\delta, 1, C)$-set with $C \sim 1$. So, let $r \geq \delta^{1 / 2}$. This time, if $B(x, r) \cap B^{\prime} \neq \emptyset$ for some ball $B^{\prime} \in \mathcal{B}_{j}^{G}$, then $r$ is large enough to ensure that $B^{\prime} \subset B(x, 2 r)$. Hence, by $\left|P \cap B^{\prime}\right| \lesssim \delta^{-1 / 2}$ for $B^{\prime} \in \mathcal{B}_{j}^{G}$, and (3.9), and the disjointness of the balls in $\mathcal{B}_{j}^{G}$, one obtains

$$
|P \cap B(x, r)| \lesssim \delta^{-1 / 2} \sum_{\substack{B^{\prime} \in \mathcal{B}_{j}^{G} \\ B^{\prime} \cap B(x, r) \neq \emptyset}} \frac{\mu\left(B^{\prime}\right)}{\mu\left(B^{\prime}\right)} \lesssim \log \delta^{-1} \sum_{\substack{B^{\prime} \in \mathcal{B}_{j}^{G} \\ B^{\prime} \subset B(x, 2 r)}} \mu\left(B^{\prime}\right) \leq \frac{\mu(B(x, 2 r))}{\delta} \lesssim \frac{r}{\delta} .
$$

I recap the main achievements so far. For a suitable scale $\delta \leq \delta_{0}^{2}$, a $(\delta, 1, C)$-set $P \subset$ $K \in\left\{K_{\pi}, P_{\mathcal{L}}\right\}$ has now been constructed with $C \sim_{\log } 1$, along with a family of $\delta^{1 / 2}$-balls $\mathcal{B}$ such that

(P1) $|P| \sim_{\log } \delta^{-1}$, and $|\mathcal{B}| \lesssim \delta^{-1 / 2}$,

(P2) $P \subset \bigcup_{B \in \mathcal{B}} B$.

3.2. Finding $\delta$-tubes. Next, relying on the counter assumptions (3.1) and (3.2), I will accompany $P$ with a finite family of $\delta$-tubes. For technical reasons, I will consider two types of $\delta$-tubes in this paper: the ordinary ones, which are $(\delta / 2)$-neighbourhoods of lines in $\mathbb{R}^{2}$, and then the dyadic ones, which I now proceed to define:

Definition 3.11 (Dyadic tubes). For $\delta=2^{-k}, k \geq 0$, a dyadic $\delta$-tube is a set of the form $\mathcal{D}(Q)$, where $Q \subset[0,1)^{2}$ is a dyadic square of side-length $\delta$, and $\mathcal{D}$ is the point-line duality mapping from Definition 2.3. One should view $\mathcal{D}(Q)$ here as a set of points in $\mathbb{R}^{2}$, not as a family of lines, see Remark 2.4. The slope of a dyadic $\delta$-tube $T=\mathcal{D}([a+\delta) \times[b+\delta))$ is defined fo be $s(T):=a$ (which is the actual slope of the line $\mathcal{D}(a, b) \subset T$ ).

The definition above is convenient for the reason that dyadic tubes have a dyadic structure (unlike ordinary tubes). For dyadic numbers $0<\delta_{1}<\delta_{2}<1$, the $\delta_{2}$-parent of a $\delta_{1}$-tube $T_{1}=\mathcal{D}\left(Q_{1}\right)$ is the unique tube $T_{2}=\mathcal{D}\left(Q_{2}\right)$ such that $Q_{2}$ is a dyadic square of side-length $\delta_{2}$ containing $Q_{1}$. The $\delta_{1}$-children of a $\delta_{2}$-tube are defined in the obvious way: note that a $\delta_{1}$-tube $T_{1}$ is the child of a $\delta_{2}$-tube $T_{2}$, if and only if $T_{1} \subset T_{2}$. Given a collection of $\delta_{1}$-tubes $\mathcal{T}$, I write $N\left(\mathcal{T}, \delta_{2}\right)$ for the cardinality of the family of $\delta_{2}$-parents of the tubes in $\mathcal{T}$ (that is, minimal family of $\delta_{2}$-tubes containing all the tubes in $\mathcal{T}$ ).

From geometric intents and purposes, dyadic tubes are locally very similar to ordinary tubes: if $T$ is a dyadic $\delta$-tube, then the intersection $T \cap B(0, R)$ is contained in an ordinary $C_{R} \delta$-tube with the same slope, for some constant $C_{R} \geq 1$ depending only on $R$. Conversely, an ordinary $\delta$-tube can be covered by a bounded number of dyadic $\delta$-tubes with nearly the same slope (with an error of $\lesssim \delta$ ). 
Assume that $\mathcal{T}_{p}$ is a collection of dyadic $\delta$-tubes, each containing a point $p \in \mathbb{R}^{2}$, and let $0<s<1$. Then $\mathcal{T}_{p}$ is called a $(\delta, s, C)$-set, if the set of slopes $s\left(\mathcal{T}_{p}\right):=\left\{s(T): T \in \mathcal{T}_{p}\right\}$ is a $(\delta, s, C)$-subset of $\mathbb{R}$. The same definition is used, if $p$ is a $\delta$-ball instead of a point, and "containing $p$ " is replaced by "intersecting $p$ ".

Similarly, a family of ordinary $\delta$-tubes, all containing a common point, is called a $(\delta, s, C)$-set, if the directions of the tubes (on $\left.S^{1}\right)$ form a $(\delta, s, C)$-set.

As stated above Definition 3.11, the plan is to use the counter assumptions (3.1) and (3.2) to accompany $P$ with a finite family of dyadic $\delta$-tubes $\mathcal{T}$. Finding $\mathcal{T}$ is a somewhat lengthy task, so I start by clarifying: what exactly is required of these tubes to end up with a contradiction? In brief, the tubes need violate the next theorem:

Theorem 3.12. Given $0<s<1$, there exists an $\epsilon=\epsilon(s)>0$ such that the following holds for small enough dyadic numbers $\delta>0$ (depending only on $s$ ). Assume that $P \subset B(0,1) \subset \mathbb{R}^{2}$ is a $\left(\delta, 1, \delta^{-\epsilon}\right)$-set with cardinality $|P| \geq \delta^{-1+\epsilon}$ and assume that

$$
N\left(P, \delta^{1 / 2}\right) \leq \delta^{-1 / 2-\epsilon} .
$$

Assume that $\mathcal{T}$ is a collection of dyadic $\delta$-tubes such that for every $p \in P$, there exists a sub-family $\mathcal{T}_{p} \subset\{T \in \mathcal{T}: p \in T\}$, which is a $\left(\delta, s, \delta^{-\epsilon}\right)$-set of cardinality $\left|\mathcal{T}_{p}\right| \geq \delta^{-s+\epsilon}$. Then either

$$
|\mathcal{T}| \geq \delta^{-2 s-\epsilon} \quad \text { or } \quad N\left(\mathcal{T}, \delta^{1 / 2}\right) \geq \delta^{-s-\epsilon} .
$$

Note that the assumptions of Theorem 3.12, in particular (3.13), are valid for the set $P$ constructed earlier, by properties (P1)-(P2). So, to prove Theorems 2.6 and 2.8, it remains to use (3.1) and (3.2) to find a family of dyadic $\delta$-tubes $\mathcal{T}$ which violates Theorem 3.12. I first need to record a few easy geometric lemmas about points and dyadic $\delta$-tubes:

Lemma 3.15. Assume that $\mathcal{T}_{p}$ is a collection of dyadic $\delta$-tubes, each containing a point $p \in$ $B(0,1)$. Then $\left|s\left(\mathcal{T}_{p}\right)\right| \sim\left|\mathcal{T}_{p}\right|$.

Proof. Write $S:=s\left(\mathcal{T}_{p}\right)$. Clearly $|S| \leq\left|\mathcal{T}_{p}\right|$, so it suffices to prove that $\left|\mathcal{T}_{p}\right| \lesssim|S|$. To this end, I will show that only four tubes in $\mathcal{T}_{p}$ can share a common slope. Assume that $a \in S$, and $T_{1}=\mathcal{D}\left([a, a+\delta) \times\left[b_{1}, b_{1}+\delta\right)\right)$ and $T_{2}=\mathcal{D}\left([a, a+\delta) \times\left[b_{2}, b_{2}+\delta\right)\right)$ both belong to $\mathcal{T}_{p}$, so that $p=\left(p_{x}, p_{y}\right) \in T_{1} \cap T_{2}$. By definition of $T_{1}, T_{2}$, this means that there exist numbers $a^{\prime}, b_{1}^{\prime}, a^{\prime \prime}, b_{2}^{\prime \prime}$ with $\max \left\{\left|a^{\prime}-a\right|,\left|a^{\prime \prime}-a\right|,\left|b_{1}^{\prime}-b_{1}\right|,\left|b_{2}^{\prime \prime}-b_{2}\right|\right\}<\delta$ such that

$$
p_{y}=a^{\prime} p_{x}+b_{1}^{\prime} \text { and } p_{y}=a^{\prime \prime} p_{x}=b_{2}^{\prime \prime} \text {. }
$$

It follows that $\left|b_{1}-b_{2}\right| \leq\left|p_{x}\right|\left|a^{\prime}-a^{\prime \prime}\right|+2 \delta<4 \delta$. This completes the proof.

Lemma 3.16. Assume that $0<\delta_{1}<\delta_{2}$ are dyadic numbers, $p \in B(0,1)$, and $T_{0}=\mathcal{D}\left(Q_{0}\right)=$ $\mathcal{D}\left(\left[a, a+\delta_{2}\right) \times\left[b, b+\delta_{2}\right)\right)$ is a dyadic $\delta_{2}$-tube. Further, assume that $\mathcal{T}_{p}$ is a $\left(\delta_{1}, s, C\right)$-set of dyadic $\delta_{1}$-tubes $T$ with $p \in T \subset T_{0}$. Then $\left|\mathcal{T}_{p}\right| \lesssim C\left(\delta_{2} / \delta_{1}\right)^{s}$.

Proof. Let $\mathcal{Q}_{p}$ be the collection of dyadic $\delta_{1}$-squares such that $\mathcal{T}_{p}=\left\{\mathcal{D}(Q): Q \in \mathcal{Q}_{p}\right\}$. Then $Q \subset Q_{0}, Q \in \mathcal{Q}_{p}$, by the assumption $T \subset T_{0}, T \in \mathcal{T}_{p}$. Hence $s\left(\mathcal{T}_{p}\right)$ is a $\left(\delta_{1}, s, C\right)$ subset of $\left[a, a+\delta_{2}\right)$, and consequently $\left|s\left(\mathcal{T}_{p}\right)\right| \leq C\left(\delta_{2} / \delta_{1}\right)^{s}$. The previous lemma completes the proof.

Lemma 3.17. Assume that $P \subset B(0,1)$ is a set and $0<\delta_{1} \leq \delta_{2}$ are dyadic numbers. Assume that $a_{2} \in \delta_{2} \mathbb{Z}$, and $P$ can be covered by $M \in \mathbb{N}$ dyadic $\delta_{2}$-tubes $\mathcal{D}\left(\left[a_{2}, a_{2}+\delta_{2}\right) \times\left[b_{j}, b_{j}+\delta_{2}\right)\right)$ with fixed slope $a_{2}$. Then the collection of all dyadic $\delta_{1}$-tubes, which intersect $P$ and have slope in $\left[a_{2}, a_{2}+\delta_{2}\right)$, can be covered by $\lesssim M$ dyadic $\delta_{2}$-tubes with slope $a_{2}$. 
Proof. Assume that $\mathcal{D}\left(\left[a_{1}, a_{1}+\delta_{1}\right) \times\left[b_{1}, b_{1}+\delta_{1}\right)\right)$ is a $\delta_{1}$-tube with slope $a_{1} \in\left[a_{2}, a_{2}+\delta_{2}\right)$, which intersects $P$ at a point $p=\left(p_{x}, p_{y}\right)$. This means that $p_{y}=a_{1}^{\prime} p_{x}+b_{1}^{\prime}$ for some $\left|a_{1}^{\prime}-a_{1}\right|<\delta_{1}$, and consequently $\left|a_{1}^{\prime}-a_{2}\right| \leq 2 \delta_{2}$. By assumption, $p$ is also covered by one of the dyadic $\delta_{2}$-tubes $\mathcal{D}\left(\left[a_{2}, a_{2}+\delta_{2}\right) \times\left[b_{j}, b_{j}+\delta_{2}\right)\right), 1 \leq j \leq M$, which implies that $p_{y}=a_{2}^{\prime} p_{x}+b_{j}^{\prime}$ for some $\left|a_{2}^{\prime}-a_{2}\right|<\delta_{2}$ and $\left|b_{j}^{\prime}-b_{j}\right|<\delta_{2}$. It follows that

$$
\left|b_{1}^{\prime}-b_{j}^{\prime}\right|=\left|p_{x}\right|\left|a_{1}^{\prime}-a_{2}^{\prime}\right| \leq 3 \delta_{2},
$$

and consequently $\left|b_{1}-b_{j}\right| \leq 5 \delta_{2}$. Now, the $\delta_{2}$-tubes of the form $\mathcal{D}\left(\left[a_{2}, a_{2}+\delta\right) \times\left[b, b+\delta_{2}\right)\right)$ with $\left|b-b_{j}\right| \leq 5 \delta$ for some $1 \leq j \leq M$, form the desired cover.

Now, finally, everything is set up to accompany $P$ with a family of dyadic $\delta$-tubes $\mathcal{T}$. The process is slightly different in the cases $K=K_{\pi}$ and $K=P_{\mathcal{L}}$.

3.2.1. The case $P \subset K=K_{\pi}$. Here we use the counter assumption (3.2), restated below:

$$
N\left(\pi_{e}\left(K_{\pi}\right), \delta\right) \leq \delta^{-s-\epsilon}, \quad e \in E, 0<\delta \leq \delta_{0},
$$

where $\mathcal{H}^{s}(E)>0$. The plan is to find a $(\delta, s)$-subset $E^{\prime} \subset E$ of near-maximal cardinality and use (3.18) to cover $P \subset K_{\pi}$ by a small family of dyadic $\delta$-tubes $\mathcal{T}_{e}$ (nearly) perpendicular to $e$ for every $e \in E^{\prime}$.

Recall that both $\delta$ and $\delta^{1 / 2}$ are dyadic numbers. Let $E_{\delta} \subset \delta \mathbb{Z}$ be a $(\delta, s, C)$-set of slopes almost perpendicular to the vectors in $E$ : more precisely, if $\theta \in E_{\delta}$, the requirement is that any line with slope $\theta$ is perpendicular to some vector in $E(10 \delta)$ (the $10 \delta$-neighbourhood is only needed to facilitate $E_{\delta} \subset \delta \mathbb{Z}$ ). By Proposition 3.3 applied to $E$ and some easy tinkering, one can choose $E_{\delta}$ with $\left|E_{\delta}\right| \sim \delta^{-s}$ (the implicit constants naturally depend on $\mathcal{H}_{\infty}^{s}(E)>0$, which will be treated as an absolute constant). Inequality (3.18) then implies that for every $\theta \in E_{\delta}$, the set $K_{\pi}$ can be covered by $\lesssim \delta^{-s-\epsilon}$ dyadic $\delta$-tubes $\mathcal{T}_{\theta}$ with slope $\theta$. Without loss of generality, all tubes will be assumed to intersect $K_{\pi}$. Let

$$
\mathcal{T}:=\bigcup_{\theta \in E_{\delta}} \mathcal{T}_{\theta} .
$$

Then $\mathcal{T}$ is a collection of dyadic $\delta$-tubes such that

(T1) $|\mathcal{T}| \lesssim \delta^{-2 s-\epsilon}$,

(T2) for every point $p \in P \subset K$, there is a $(\delta, s, C)$-subset $\mathcal{T}_{p} \subset\{T \in \mathcal{T}: p \in T\}$ of cardinality $\left|\mathcal{T}_{p}\right| \sim \delta^{-s}$

(T3) $N\left(\mathcal{T}, \delta^{1 / 2}\right) \lesssim \delta^{-s-\epsilon}$.

Here (T1) follows from $\left|E_{\delta}\right| \sim \delta^{-s}$ and $\left|\mathcal{T}_{\theta}\right| \lesssim \delta^{-s-\epsilon}$. To see the (T2), fix $p \in P \subset K=K_{\pi}$ and observe that $p \in T$ for some tube $T \in \mathcal{T}_{\theta}$, for every $\theta \in E_{\delta}$. The family $\mathcal{T}_{p}$ can be picked among those tubes $T$. The the claim (T3) requires a bit of extra work. I first recall the following basic estimate:

Proposition 3.19. Assume that $K \subset B(0,1)$ with $\mathcal{H}^{1}(K)>0$ and $0<t<1$. Then

$$
N\left(\left\{e \in S^{1}: N\left(\pi_{e}(K), \delta\right) \leq \delta^{-t}\right\}, \delta\right) \lesssim \log \delta^{-t},
$$

where the implicit constants depend on $\mathcal{H}^{1}(K)$.

This is, for instance, inequality (1.2) in [18], and the proof can be found on p. 9 of the same paper. The proposition also easily follows from Lemma 4.1 below. Now, apply 
Proposition 3.19 with $t=s+\epsilon$, at scale $\delta^{1 / 2}$, and the set $K_{\pi}$. The conclusion is that

$$
N\left(E_{\delta}, \delta^{1 / 2}\right) \lesssim N\left(\left\{e \in S^{1}: N\left(\pi_{e}\left(K_{\pi}\right), \delta^{1 / 2}\right) \leq C \delta^{-(s+\epsilon) / 2}\right\}, \delta^{1 / 2}\right) \lesssim \log \delta^{-(s+\epsilon) / 2} .
$$

The first inequality follows from the fact that if $\theta \in E_{\delta}\left(\delta^{1 / 2}\right)$, then a unit vector $e_{\theta}$ parallel to a line with slope $\theta$ lies at distance $\lesssim \delta^{1 / 2}$ from some vector $e \in E^{\perp}$. Then, if $e_{\theta}^{\perp}, e^{\perp}$ are perpendicular to $e_{\theta}, e$, one has $\left|e_{\theta}^{\perp}-e^{\perp}\right| \lesssim \delta^{1 / 2}$, and

$$
N\left(\pi_{e_{\theta}^{\perp}}\left(K_{\pi}\right), \delta^{1 / 2}\right) \lesssim N\left(\pi_{e^{\perp}}\left(K_{\pi}\right), \delta^{1 / 2}\right) \leq \delta^{-(s+\epsilon) / 2}
$$

by the definition of $E$ (and $\delta^{1 / 2} \leq \delta_{0}$ ). This means that $e_{\theta}^{\perp}$ belongs to the set in the middle of (3.20) for large enough $C \geq 1$, which implies the first inequality of (3.20) for the vectors $e_{\theta}, \theta \in E_{\delta}-$ and then for the points $\theta \in E_{\delta}$.

With (3.20) in hand, pick a collection $\left\{\theta_{j}\right\}_{j \in \mathcal{J}} \subset \delta^{1 / 2} \mathbb{Z}$ such that $|\mathcal{J}| \lesssim \delta^{-(s+\epsilon) / 2}$ and $E_{\delta}$ is contained in the union of the dyadic intervals $\left[\theta_{j}, \theta_{j}+\delta^{1 / 2}\right)$. The numbers $\theta_{j}$ can be picked at distance $\leq \delta^{1 / 2}$ from some point in $E_{\delta}$, so (3.21) applies with $\theta=\theta_{j}$ : the conclusion is that $K_{\pi}$ can be covered by $\lesssim \delta^{-(s+\epsilon) / 2}$ dyadic $\delta^{1 / 2}$-tubes with slope $\theta_{j}$. Consequently, by Lemma 3.17, all the tubes in the families $\mathcal{T}_{\theta}$, with $\theta \in E_{\delta} \cap\left[\theta_{j}, \theta_{j}+\delta^{1 / 2}\right)$, can be covered by $\lesssim \delta^{-(s+\epsilon) / 2}$ dyadic $\delta^{1 / 2}$-tubes of slope $\theta_{j}$. It follows that all the tubes in $\mathcal{T}$ can be covered by $\lesssim \delta^{-(s+\epsilon) / 2} \cdot \delta^{-(s+\epsilon) / 2}=\delta^{-s-\epsilon}$ dyadic $\delta^{1 / 2}$-tubes, as claimed in (T3).

3.2.2. The case $P \subset K=P_{\mathcal{L}}$. This case is simpler: again, the aim is to find a family of dyadic $\delta$-tubes $\mathcal{T}$ satisfying the conditions (T1)-(T3). Recall the main counter assumption (3.1):

$$
N\left(K_{F}, \delta\right) \leq \delta^{-2 s-\epsilon}, \quad 0<\delta \leq \delta_{0} .
$$

Also, recall that $\mathcal{L}$ is a compact set of lines $L$, which form a large angle with the $y$-axis, and with the property that $\mathcal{H}_{\infty}^{s}\left(K_{F} \cap L\right) \geq c>0$. By (P1), $P \subset P_{\mathcal{L}}$ is a $(\delta, 1, C)$-set with $C \sim_{\log } 1$ of cardinality $|P| \sim_{\log } \delta^{-1}$.

I record a small observation about the point-line duality:

Lemma 3.23. Assume that $(c, d) \in \mathcal{D}(a, b)$. Then $(a, b) \in \mathcal{D}(-c, d)$.

Proof. By assumption $d=a c+b$, or $b=(-c) a+d$. Hence $(a, b) \in \mathcal{D}(-c, d)$.

Note that $L_{p}:=\mathcal{D}(p) \in \mathcal{L}$ for all $p \in P \subset P_{\mathcal{L}}$. It follows from $\mathcal{H}_{\infty}^{s}\left(K_{F} \cap L_{p}\right) \geq c$ and Proposition 3.3 that $K_{F} \cap L_{p}$ contains a $(\delta, s, C)$-set with $C \sim 1$ and cardinality $\sim \delta^{-s}$ (I treat $c$ as an absolute constant). Let $\mathcal{Q}_{p}^{\prime}=\left\{\left(a_{i}, a_{i}+\delta\right] \times\left[b_{i}, b_{i}+\delta\right)\right\}_{i \in \mathcal{I}}$ be the collection of (not quite dyadic) $\delta$-squares of the form $(a, a+\delta] \times[b, b+\delta)$ with $a, b \in \delta \mathbb{Z}$, which contain a point in the said $(\delta, s, C)$-set on $K_{F} \cap L_{p}$. Then $\mathcal{Q}_{p}:=\left\{\left[-a_{i}-\delta, a_{i}\right) \times\left[b_{i}, b_{i}+\delta\right)\right\}_{i \in \mathcal{I}}$ is a collection of dyadic squares. Since $L_{p}$ is quantitatively non-vertical, the numbers $a_{i}$ form a $(\delta, s, C)$-set, and hence $\mathcal{T}_{p}:=\left\{\mathcal{D}(Q): Q \in \mathcal{Q}_{p}\right\}$ is a $(\delta, s, C)$-set of dyadic $\delta$-tubes by definition. Each tube $\mathcal{D}\left(\left[-a_{i}-\delta, a_{i}\right) \times\left[b_{i}, b_{i}+\delta\right)\right) \in \mathcal{T}_{p}$ moreover contains $p$, since $\left(a_{i}, a_{i}+\delta\right] \times\left[b_{i}, b_{i}+\delta\right)$ contains a point $\left(x_{1}, x_{2}\right) \in L_{p}=\mathcal{D}(p)$ by definition, and then

$$
p \in \mathcal{D}\left(-x_{1}, x_{2}\right) \in \mathcal{D}\left(\left[-a_{i}-\delta, a_{i}\right) \times\left[b_{i}, b_{i}+\delta\right)\right)
$$

by Lemma 3.23. Writing

$$
\mathcal{T}:=\bigcup_{p \in P} \mathcal{T}_{p}
$$


the condition (T2) is automatically valid. Note that $\mathcal{T}$ consists of $\mathcal{D}$-images of $\delta$-squares $Q$ meeting $\left\{(-x, y):(x, y) \in K_{F}\right\}$. Hence $|\mathcal{T}| \lesssim \delta^{-2 s-\epsilon}$ by (3.22), which gives (T1). Finally, all these $\delta$-squares can be covered by $\lesssim \delta^{-s-\epsilon / 2}$ dyadic squares of side-length $\delta^{1 / 2}$, by (3.22) at scale $\delta^{1 / 2}$. This gives $N\left(\mathcal{T}, \delta^{1 / 2}\right) \lesssim \delta^{-s-\epsilon / 2}$, which is a little bit better than (T3).

3.3. Concluding the proofs of Theorems 2.6 and 2.8. In both cases $K=K_{\pi}$ and $K=\mathcal{P}_{\mathcal{L}}$, a finite set $P$ and a collection of tubes $\mathcal{T}$ have now been found, satisfying (P1)-(P2) and (T1)-(T3), respectively. The counter assumptions (3.1) and (3.2) were heavily used. Now, to conclude the proofs of both Theorems 2.6 and 2.8, it suffices to show that $P$ can $\mathcal{T}$ cannot exist, for sufficiently small $\epsilon>0$. This follows from Theorem 3.12. Indeed, we already observed earlier that $P$ satisfies the hypotheses of Theorem 3.12, by (P1)-(P2). Moreover, the condition (T2) is even slightly better than what Theorem 3.12 requires from $\mathcal{T}$, and conditions (T1) and (T3) literally state that the main conclusion (3.14) of Theorem 3.12 fails (assuming that $\epsilon>0$ is sufficiently small). So, a contradiction has been reached, and the proofs of Theorems 2.6 and 2.8 are complete.

\section{AN INCIDENCE BOUND FOR POINTS AND TUBES}

The purpose of this section is to prove Theorem 3.12, stated below as Theorem 4.3 for convenience. I start with a simple - and well-known - incidence bound: heuristically, Theorem 4.3 can then be viewed as an $\epsilon$-improvement of this "trivial" bound, although the hypotheses are somewhat stronger.

Lemma 4.1. Let $0<s<1$ and assume that $\epsilon>0$ is small enough (depending on $s$ only). Assume that $P \subset B(0,1) \subset \mathbb{R}^{2}$ is a $\left(\delta, 1, \delta^{-\epsilon}\right)$-set with $|P| \geq \delta^{-1+\epsilon}$, and $\mathcal{T}$ is a family of dyadic $\delta$-tubes. Assume that for every point $p \in P$, there exists a sub-family $\mathcal{T}_{p} \subset\{T \in \mathcal{T}: p \in T\}$ with $\left|\mathcal{T}_{p}\right| \geq \delta^{-s+\epsilon}$. Then $|\mathcal{T}| \gtrsim \log \delta^{-2 s+6 \epsilon}$.

Proof. Define the set of incidences as follows:

$$
I(P, \mathcal{T}):=\left\{(p, T) \in P \times \mathcal{T}: T \in \mathcal{T}_{p}\right\} .
$$

Note the slightly non-standard definition: the condition $p \in T$ is necessary, but not sufficient, for $(p, T) \in I(P, \mathcal{T})$. Evidently

$$
|I(P, \mathcal{T})| \geq \sum_{p \in P}\left|\mathcal{T}_{p}\right| \geq \delta^{-1-s+2 \epsilon} .
$$

Write

$$
N_{T}:=\left|\left\{p \in P: T \in \mathcal{T}_{p}\right\}\right|,
$$

and estimate $|I(P, \mathcal{T})|$ from above as follows:

$$
\begin{aligned}
|I(P, \mathcal{T})| & =\sum_{T \in \mathcal{T}} N_{T} \leq|\mathcal{T}|^{1 / 2}\left(\sum_{T \in \mathcal{T}}\left|\left\{(p, q) \in P \times P: T \in \mathcal{T}_{p} \cap \mathcal{T}_{q}\right\}\right|\right)^{1 / 2} \\
& \lesssim|\mathcal{T}|^{1 / 2}\left(\sum_{p \in P}\left|\mathcal{T}_{p}\right|\right)^{1 / 2}+|\mathcal{T}|^{1 / 2}\left(\sum_{p \neq q}\left|\mathcal{T}_{p} \cap \mathcal{T}_{q}\right|\right)^{1 / 2}
\end{aligned}
$$


The first term equals $|\mathcal{T}|^{1 / 2}|I(P, \mathcal{T})|^{1 / 2}$. So, in case the first term dominates, one obtains $|\mathcal{T}| \gtrsim|I(P, \mathcal{T})| \geq \delta^{-1-s-2 \epsilon}$, which beats the desired estimate, if $\epsilon>0$ is small enough. To estimate the second sum, one observes that

$$
\left|\mathcal{T}_{p} \cap \mathcal{T}_{q}\right| \leq\left|\left\{T \in \mathcal{T}_{p}: q \in T\right\}\right| \lesssim \frac{1}{|p-q|},
$$

using the fact that the slopes of the tubes in $\mathcal{T}_{p}$ are $\delta$-separated (first prove the inequality for any family of ordinary $\delta$-tubes, which contain $p$ and have $\delta$-separated slopes, and finally use $P \subset B(0,1)$ to reduce the dyadic case the non-dyadic one). Hence, if the second sum dominates, one obtains

$$
|I(P, \mathcal{T})| \lesssim|\mathcal{T}|^{1 / 2}\left(\sum_{p \neq q} \frac{1}{|p-q|}\right)^{1 / 2} \lesssim_{\log }|\mathcal{T}|^{1 / 2} \delta^{-1-\epsilon} .
$$

The second inequality is a standard estimate using the $\left(\delta, 1, \delta^{-\epsilon}\right)$-set hypothesis (for each point $p \in P$, divide the points $q \neq p$ into dyadic annuli around $p$, and make the obvious estimates). Consequently $|\mathcal{T}| \gtrsim_{\log } \delta^{-2 s+6 \epsilon}$, as claimed.

Remark 4.2. The lemma works verbatim the same, if the points $p$ are replaced by disjoint $\delta$-balls $B$, and $\mathcal{T}_{B}$ (instead of $\mathcal{T}_{p}$ ) consists of dyadic $\delta$-tubes meeting $B$ (instead of containing $p$ ). Instead of $|p-q|$, consider the distance between the centres of the relevant balls.

The next theorem is just Theorem 3.12 repeated for convenience:

Theorem 4.3. Given $0<s<1$, there exists an $\epsilon=\epsilon(s)>0$ such that the following holds for small enough dyadic numbers $\delta>0$ (depending only on $s$ ). Assume that $P \subset B(0,1) \subset \mathbb{R}^{2}$ is a $\left(\delta, 1, \delta^{-\epsilon}\right)$-set with cardinality $|P| \geq \delta^{-1+\epsilon}$ and assume that

$$
N\left(P, \delta^{1 / 2}\right) \leq \delta^{-1 / 2-\epsilon} .
$$

Assume that $\mathcal{T}$ is a collection of dyadic $\delta$-tubes such that for every $p \in P$, there exists a sub-family $\mathcal{T}_{p} \subset\{T \in \mathcal{T}: p \in T\}$, which is a $\left(\delta, s, \delta^{-\epsilon}\right)$-set of cardinality $\left|\mathcal{T}_{p}\right| \geq \delta^{-s+\epsilon}$. Then either

$$
|\mathcal{T}| \geq \delta^{-2 s-\epsilon} \quad \text { or } \quad N\left(\mathcal{T}, \delta^{1 / 2}\right) \geq \delta^{-s-\epsilon} .
$$

Remark 4.6. Without loss of generality, one may clearly assume that

$$
\mathcal{T}=\bigcup_{p \in P} \mathcal{T}_{p}
$$

However, it is good to keep in mind that $p \in T \in \mathcal{T}$ can, nevertheless, happen for some tubes $T \notin \mathcal{T}_{p}$. It would make life somewhat easier, if one could assume

$$
\text { " } T \in \mathcal{T}_{p} \Longleftrightarrow p \in T ",
$$

but I do not know how to make such a reduction.

Remark 4.7. I suspect that the assumptions of Theorem 4.3 are unnecessarily strong. The following conjecture seems plausible. Let $0<s<1$ and $s \leq \tau<1$. Assume that $P$ is a $\left(\delta, 1, \delta^{-\epsilon}\right)$-set with $|P| \geq \delta^{-1+\epsilon}$, and assume that $\mathcal{T}$ is a family of (dyadic) $\delta$-tubes such that, for every $p \in P$, the sub-family $\{T \in \mathcal{T}: p \in T\}$ contains a $\left(\delta, \tau, \delta^{-\epsilon}\right)$-set $\mathcal{T}_{p}$ of cardinality $\left|\mathcal{T}_{p}\right| \geq \delta^{-s+\epsilon}$. Then $|\mathcal{T}| \geq \delta^{-2 s-\epsilon}$, if $\epsilon>0$ is small enough in a manner depending only on $s$ and $\tau$. This would give an improvement for the lower Minkowski dimension - and possibly even Hausdorff dimension - of Furstenberg sets. 
4.1. The main counter assumption. The proof of Theorem 4.3 begins, and I make a counter assumption:

$$
\delta^{-2 s+6 \epsilon} \lesssim \log |\mathcal{T}| \leq \delta^{-2 s-\epsilon} \quad \text { and } \quad N\left(\mathcal{T}, \delta^{1 / 2}\right) \leq \delta^{-s-\epsilon} .
$$

(The lower bound for $|\mathcal{T}|$ is simply a consequence of Lemma 4.1.) In the sequel, I will constantly use the notations $A \lesssim B, A \gtrsim B$ and $A \approx B$ to signify equivalence up to a factor of $C_{\epsilon} \delta^{-C \epsilon}$, where $\epsilon>0$ is the counter assumption parameter from (4.8). So, for instance $A \lesssim B$ means that $A \leq C_{\epsilon} \delta^{-C \epsilon} B$ for some constant $C \geq 1$ depending only on $s$, and some constant $C_{\epsilon} \geq 1$ depending only on $\epsilon$ and $s$. It is also convenient to define that a finite collection of points or $\delta$-balls is a $(\delta, t)$-set, if it is a $\left(\delta, t, C_{\epsilon} \delta^{-C \epsilon}\right)$-set for constants $C, C_{\epsilon} \geq 1$ as above.

In brief, the proof below shows that, under the counter assumption (4.8), certain quantities $A, B$ satisfy $A \lesssim B$. However, Proposition 4.36 below states that $A \geq \delta^{-\epsilon_{s}} B$ for some $\epsilon_{s}>0$ depending only on $s$. Consequently, the counter assumption cannot hold for arbitrarily small $\epsilon>0$, and Theorem 4.3 follows.

Before starting in earnest, I gather a list of notation which will be introduced more carefully during the proof:

$B, \mathcal{B}$ The letter $B$ stands for a ball of radius $\delta^{1 / 2}$, and $\mathcal{B}$ stands for a family of $\delta^{1 / 2}$-balls, see the start of Section 4.2.

$P$ The letter $P$ stands for the $\delta$-separated set from the hypothesis of Theorem 4.3.

$\mathcal{T}, \mathcal{T}_{\delta^{1 / 2}}$ The letter $\mathcal{T}$ stands for the family of $\delta$-tubes from Theorem 4.3 , whereas $\mathcal{T}_{\delta^{1 / 2}}$ is a cover of the tubes in $\mathcal{T}$ by $\delta^{1 / 2}$-tubes; see the definition above (4.11).

' Apostrophes generally mean refinements: $P^{\prime}, \mathcal{T}^{\prime}, \mathcal{B}^{\prime}$ are large subsets of $P, \mathcal{T}, \mathcal{B}$.

$\mathcal{T}_{p}, \mathcal{T}_{\delta^{1 / 2}}^{B}$ Subsets of $\mathcal{T}$ or $\mathcal{T}_{\delta^{1 / 2}}$ containing a fixed point $p$, or intersecting a fixed ball $B$, are denoted by $\mathcal{T}_{p}$ or $\mathcal{T}_{\delta^{1 / 2}}^{B}$; see the line after (4.11), and the hypothesis of Theorem 4.3.

$P_{B}, p_{B}, P_{\delta^{1 / 2}}$ A certain subset of $P \cap B$ is denoted by $P_{B}$. The notation $p_{B}$ stands for a point in $P \cap B$ (not necessarily in $P_{B}$ ), which has been "singled-out". For both the definitions, see the lines after (4.16). The union of the points $p_{B}$, over all the balls $B \in \mathcal{B}$, is denoted by $P_{\delta^{1 / 2}}$, see (4.23).

$M_{T}, N_{T}$ The letter $M_{T}$ stands for the number of balls $B \in \mathcal{B}$ intersecting the $\delta^{1 / 2}$-tubes $T$. Similarly, $N_{T}$ stands for the number of points in $P$ contained in a $\delta$-tube $T$. See the lines after (4.27) and Lemma 4.29 for definitions.

$\mathcal{T}_{0}, \mathcal{B}_{0}$ The letter $\mathcal{T}_{0}$ stands for a sub-family of $\mathcal{T}$, consisting of $\delta$-tubes contained in a fixed $\delta^{1 / 2}$-tube $T_{0}$. The family $\mathcal{B}_{0}$ consists of balls in $\mathcal{B}$ meeting the same tube $T_{0}$. See (4.34) and below.

4.2. Considerations at scale $\delta^{1 / 2}$. Let $\mathcal{B}$ be a collection of $\delta^{1 / 2}$-balls covering $P$. Then $|\mathcal{B}| \lesssim \delta^{-1 / 2}$ by the assumption (4.4), but clearly also $|\mathcal{B}| \gtrsim \delta^{-1 / 2}$, since $P$ is a $(\delta, 1)$-set with $|P| \approx \delta^{-1}$. Moreover, since $|P \cap B| \lesssim \delta^{-1 / 2}$ for every $B \in \mathcal{B}$ by the $(\delta, 1)$-set assumption, and $|P| \approx \delta^{-1}$, one sees that a subset $P^{\prime} \subset P$ with $\left|P^{\prime}\right| \approx \delta^{-1}$ is covered by balls $B \in \mathcal{B}$ with

$$
|P \cap B| \approx \delta^{-1 / 2} .
$$

Since $P^{\prime}$ satisfies all the same assumptions as $P$ - with slightly worse constants perhaps - I may and will assume that $P^{\prime}=P$; thus, one may assume that (4.9) holds for all balls $B \in \mathcal{B}$. By throwing away an additional fraction of the points in $P$, one can assume that 
the balls in $\mathcal{B}$ are $\delta^{1 / 2}$-separated:

$$
\operatorname{dist}\left(B, B^{\prime}\right) \geq \delta^{1 / 2}, \quad B, B^{\prime} \in \mathcal{B} .
$$

(In the most relevant application of Theorem 4.3 , to the set $P$ from the previous section, both (4.9) and (4.10) are a priori guaranteed by (3.10) and the construction of $P$ below (3.10)). Furthermore, (4.9) implies that $\mathcal{B}$ is a $\left(\delta^{1 / 2}, 1\right)$-set: if the midpoints of the balls $B \in \mathcal{B}$ are temporarily denoted by $R$, then

$$
\frac{r}{\delta} \gtrsim|P \cap B(x, 2 r)| \gtrsim|R \cap B(x, r)| \cdot \delta^{-1 / 2}, \quad x \in \mathbb{R}^{2}, r \geq \delta^{1 / 2} .
$$

Now, let $\mathcal{T}_{\delta^{1 / 2}}$ be a collection of $\delta^{1 / 2}$-parents of the tubes in $\mathcal{T}$. By the counter assumption (4.8),

$$
\left|\mathcal{T}_{\delta^{1 / 2}}\right| \lesssim \delta^{-s}
$$

For $B \in \mathcal{B}$, let $\mathcal{T}_{\delta^{1 / 2}}^{B}$ be the collection of tubes in $\mathcal{T}_{\delta^{1 / 2}}$ intersecting $B$. Pick a large constant $C \geq 1$. I now claim that at most half of the balls $B \in \mathcal{B}$ can satisfy

$$
\left|\mathcal{T}_{\delta^{1 / 2}}^{B}\right| \geq \delta^{-s / 2-C \epsilon} .
$$

Indeed, since the collection of balls $\mathcal{B}$ is a $\left(\delta^{1 / 2}, 1\right)$-set, Lemma 4.1 applies at scale $\delta^{1 / 2}$ (see also Remark 4.2). The conclusion is that if $|\mathcal{B}| / 2 \approx \delta^{-1 / 2}$ balls in $\mathcal{B}$ did satisfy $\left|\mathcal{T}_{\delta^{1 / 2}}^{B}\right| \geq$ $\delta^{-s / 2-C \epsilon}$ the inequality above, then $\left|\mathcal{T}_{\delta^{1 / 2}}\right| \gtrsim \delta^{-s-C \epsilon+6 \epsilon}$. For $C \geq 1$ large enough, this would contradict (4.11).

I now discard all the balls from $\mathcal{B}$ with $\left|\mathcal{T}_{\delta^{1 / 2}}^{B}\right| \geq \delta^{-s / 2-C \epsilon}$ along with the points of $P$ contained in them. Since $|\mathcal{B}| / 2 \approx \delta^{-1 / 2}$ balls remain, and each of these balls satisfies (4.9), also $\approx \delta^{-1}$ points of $P$ remain. Thus, passing to these subsets of $P$ and $\mathcal{B}$ if necessary, one may assume without loss of generality the uniform bound

$$
\left|\mathcal{T}_{\delta^{1 / 2}}^{B}\right| \lesssim \delta^{-s / 2}, \quad B \in \mathcal{B} .
$$

On the other hand, the tubes in $\mathcal{T}_{\delta^{1 / 2}}^{B}$ cover all the tubes $T \in \mathcal{T}_{p}$, for any individual $p \in B$. Since $\mathcal{T}_{p}$ is a $(\delta, s)$-set, any fixed dyadic $\delta^{1 / 2}$-tube can only cover $\lesssim \delta^{-s / 2}$ tubes $T \in \mathcal{T}_{p}$ by Lemma 3.16. Since $\left|\mathcal{T}_{p}\right| \approx \delta^{-s}$ by assumption, it follows that $\left|\mathcal{T}_{\delta^{1 / 2}}^{B}\right| \gtrsim \delta^{-s / 2}$. Combining this with (4.12), one obtains

$$
\left|\mathcal{T}_{\delta^{1 / 2}}^{B}\right| \approx \delta^{-s / 2}, \quad B \in \mathcal{B} .
$$

By the argument in the paragraph above (4.12), this implies that

$$
\left|\mathcal{T}_{\delta^{1 / 2}}\right| \approx \delta^{-s}
$$

matching the upper bound in the counter assumption (4.8). I still need to regularise the situation a little further: even though (4.13) now holds uniformly for $B \in \mathcal{B}$, it can happen that the tubes $T \in \mathcal{T}_{\delta^{1 / 2}}^{B}$ contain significantly different numbers tubes $T \in \mathcal{T}_{p}$ and even worse, these numbers can depend on the choice of $p \in B \cap P$.

To remedy this, fix $B \in \mathcal{B}$ and any $p \in B \cap P$ for the moment. Then the tubes $T \in \mathcal{T}_{p}$, are covered by the tubes in $\mathcal{T}_{\delta^{1 / 2}}^{B}$ (since $p \in T \subset T_{0} \in \mathcal{T}_{\delta^{1 / 2}}$ forces $T_{0} \cap B \neq \emptyset$ ). As already observed above, by Lemma 3.16, every tube $T \in \mathcal{T}_{\delta^{1 / 2}}^{B}$ can only have $\lesssim \delta^{-s / 2}$ children in $\mathcal{T}_{p}$. Since $\left|\mathcal{T}_{p}\right| \approx \delta^{-s}$, it follows from this and (4.13) that there necessarily exists a family

$$
\mathcal{T}_{\delta^{1 / 2}}^{B}(p) \subset \mathcal{T}_{\delta^{1 / 2}}^{B}
$$


with $\left|\mathcal{T}_{\delta^{1 / 2}}^{B}\right| \approx \delta^{-s / 2}$ such that every tube in $\mathcal{T}_{\delta^{1 / 2}}^{B}(p)$ has $\approx \delta^{-s / 2}$ children in $\mathcal{T}_{p}$. Then the family $\mathcal{T}_{\delta^{1 / 2}}^{B}(p)$ is a $\left(\delta^{1 / 2}, s\right)$-set. To see this, fix a ball $B(x, r) \subset \mathbb{R}$ with $r \geq \delta^{1 / 2}$. If $B(x, r)$ contains $s\left(T_{0}\right)$ for some $T_{0} \in \mathcal{T}_{\delta^{1 / 2}}^{B}(p)$, then $B(x, 2 r)$ contains $s(T)$ for each of the $\approx \delta^{-s / 2}$ tubes $T \in \mathcal{T}_{p}$ with $T \subset T_{0}$. There are $\approx \delta^{-s / 2}$ such slopes $s(T)$ by Lemma 3.15. Since $s\left(\mathcal{T}_{p}\right)$ is a $(\delta, s)$-set, $B(x, r)$ contains no more than $\lesssim(r / \delta)^{s}$ elements in $s\left(\mathcal{T}_{p}\right)$, and consequently no more than $\lesssim\left(r / \delta^{1 / 2}\right)^{s}$ elements in $s\left(\mathcal{T}_{\delta^{1 / 2}}^{B}(p)\right)$.

For every $p \in B \cap P$, the family $\mathcal{T}_{\delta^{1 / 2}}^{B}(p) \subset \mathcal{T}_{\delta^{1 / 2}}^{B}$ is a subset of cardinality

$$
\left|\mathcal{T}_{\delta^{1 / 2}}^{B}(p)\right| \approx\left|\mathcal{T}_{\delta^{1 / 2}}^{B}\right| \approx \delta^{-s / 2}
$$

by (4.16), and so

$$
\sum_{T \in \mathcal{T}_{\delta^{1 / 2}}^{B}}\left|\left\{p: T \in \mathcal{T}_{\delta^{1 / 2}}^{B}(p)\right\}\right|=\sum_{p \in B \cap P}\left|\mathcal{T}_{\delta^{1 / 2}}^{B}(p)\right| \gtrsim\left|B \cap P \| \mathcal{T}_{\delta^{1 / 2}}^{B}\right| .
$$

Applying Cauchy-Schwarz on the left hand side then gives

$$
\sum_{p, q \in B \cap P}\left|\mathcal{T}_{\delta^{1 / 2}}^{B}(p) \cap \mathcal{T}_{\delta^{1 / 2}}^{B}(q)\right| \gtrsim|B \cap P|^{2}\left|\mathcal{T}_{\delta^{1 / 2}}^{B}\right|,
$$

which implies that there exist $\approx|B \cap P|^{2} \approx \delta^{-1}$ pairs of points $p, q \in B \cap P$ such that

$$
\left|\mathcal{T}_{\delta^{1 / 2}}^{B}(p) \cap \mathcal{T}_{\delta^{1 / 2}}^{B}(q)\right| \approx\left|\mathcal{T}_{\delta^{1 / 2}}^{B}\right| \approx \delta^{-s / 2} .
$$

Consequently, one can fix a single point $p=p_{B} \in P \cap B$ such that (4.16) holds for $\approx \delta^{-1 / 2}$ points $q \in B \cap P$. Denote these points by $P_{B}$, so (4.16) becomes

$$
\left|\mathcal{T}_{\delta^{1 / 2}}^{B}\left(p_{B}\right) \cap \mathcal{T}_{\delta^{1 / 2}}^{B}(q)\right| \approx \delta^{-s / 2}, \quad q \in P_{B} .
$$

Now, write

$$
\mathcal{T}_{B}^{\prime \prime}:=\mathcal{T}_{\delta^{1 / 2}}^{B}\left(p_{B}\right),
$$

which is a $\left(\delta^{1 / 2}, s\right)$-set of dyadic $\delta^{1 / 2}$-tubes meeting $B$ of cardinality $\left|\mathcal{T}_{B}^{\prime \prime}\right| \approx \delta^{-s / 2}$. In fact

$$
p_{B} \in T \text { for all } T \in \mathcal{T}_{B}^{\prime \prime},
$$

since the tubes in $\mathcal{T}_{B}^{\prime \prime}$ contain some tubes in $\mathcal{T}_{p_{B}}$. There are two apostrophes in $\mathcal{T}_{B}^{\prime \prime}$, because the collection will, eventually, undergo two refinements (or "removals of exceptional sets"), and the end product will be denoted by $\mathcal{T}_{B}$.

4.3. Refining the families of $\delta^{1 / 2}$-tubes. For a tube $T=\mathcal{D}\left(\left[a, \delta^{1 / 2}\right) \times\left[b, \delta^{1 / 2}\right)\right) \in \mathcal{T}_{B}^{\prime \prime}$, let $e_{T} \in S^{1}$ for the unit vector perpendicular to line $\mathcal{D}(a, b) \subset T$, and let $E_{B}:=\left\{e_{T}: T \in\right.$ $\left.\mathcal{T}_{B}\right\}$. Then $E_{B}$ is a $\left(\delta^{1 / 2}, s\right)$-set. Denote by $\pi_{e_{T}}$ the orthogonal projection onto the line spanned by $e_{T}$, see Figure 2. Informally, the next lemma says that "for any $B \in \mathcal{B}$, in an overwhelming majority of directions $e_{T} \in E_{B}$, the set $\pi_{e_{T}}\left(P_{B}\right)$, and all its reasonably large subsets, contain a $(\delta, s)$-set of nearly maximal cardinality, namely $\approx \delta^{-s / 2}$.

Lemma 4.19. Let $C_{0}, C_{1}, C_{2} \geq 1$ be constants. Then, if $C_{2}$ is sufficiently large, depending on $C_{0}, C_{1}$ and the various constants behind the $\lesssim$-notation used above, there are at least (1$\left.\delta^{C_{1} \epsilon}\right)\left|\mathcal{T}_{B}^{\prime \prime}\right|$ "good" vectors $e_{T} \in E_{B}$ with the following property: if $P_{B}^{\prime} \subset P_{B}$ is a subset of cardinality $\left|P_{B}^{\prime}\right| \geq \delta^{C_{0} \epsilon}\left|P_{B}\right|$, then $\pi_{e_{T}}\left(P_{B}^{\prime}\right)$ contains a $\left(\delta, s, C_{2}\right)$-set of cardinality $\geq \delta^{C_{2} \epsilon-s / 2}$. 


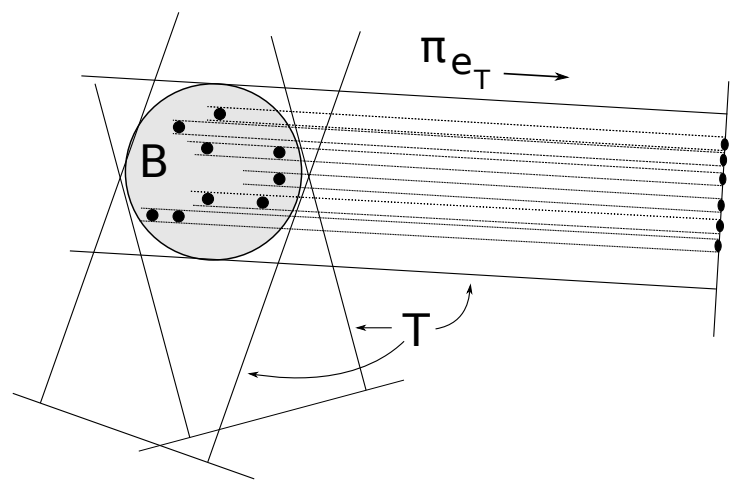

FIgURE 2. The tubes $T \in \mathcal{T}_{B}^{\prime \prime}$, and the projections $\pi_{e_{T}}$.

Proof. The proof is a variation of the standard "potential theoretic" argument, invented by Kaufman [12]; if the reader is not familiar with the technique, a similar but cleaner statement is Theorem 2 in [6]. First, observe that $\delta^{-1 / 2} P_{B}$ is a $\left(\delta^{1 / 2}, 1\right)$-set of cardinality $\approx \delta^{-1 / 2}$. Next, consider the measures

$$
\mu_{B}:=\frac{1}{\left|P_{B}\right|} \sum_{p \in \delta^{-1 / 2} P_{B}} \frac{\chi_{B\left(p, \delta^{1 / 2}\right)}}{\delta}
$$

and

$$
\nu:=\frac{1}{\left|E_{B}\right|} \sum_{e \in E_{B}} \frac{\chi_{B\left(e, \delta^{1 / 2}\right) \cap S^{1}}}{\delta^{1 / 2}}
$$

and note that $\mu_{B}\left(\mathbb{R}^{2}\right) \sim 1 \sim \nu\left(S^{1}\right)$. For $r \geq \delta^{1 / 2}$, one has the uniform estimates $\mu_{B}(B(x, r)) \lesssim r$ and $\nu(B(e, r)) \lesssim r^{s}$, while for $0<r \leq \delta^{1 / 2}$ one has the obvious improved estimates. After some straightforward computations, it follows that

$$
\int_{S^{1}} I_{s}\left(\pi_{e \sharp} \mu_{B}\right) d \nu e:=\iint\left[\int_{S^{1}} \frac{d \nu e}{\left|\pi_{e}(x)-\pi_{e}(y)\right|^{s}}\right] d \mu_{B} x d \mu_{B} y \lesssim 1 .
$$

Indeed, the inner integral (in brackets) can be estimated by $\lesssim 1 /|x-y|^{s}$, and then

$$
\int_{S^{1}} I_{s}\left(\pi_{e \sharp} \mu_{B}\right) d \nu e \lesssim \int\left[\int \frac{d \mu_{B} x}{|x-y|^{s}}\right] d \mu_{B} y \lesssim 1,
$$

since the inner integral is again bounded by $\lesssim 1$ for any $y \in \mathbb{R}^{2}$. Consequently, by Chebyshev's inequality,

$$
\nu\left(\left\{e \in S^{1}: I_{s}\left(\pi_{e \sharp} \mu_{B}\right) \geq \delta^{-C \epsilon}\right\}\right) \lesssim \delta^{C \epsilon}, \quad C \geq 1 .
$$

Now, first, choose $C_{2}^{\prime} \geq 1$ so large that $\left.\nu\left(\left\{e: I_{s}\left(\pi_{e \sharp} \mu_{B}\right\}\right) \geq \delta^{-C_{2}^{\prime} \epsilon}\right\}\right) \leq \delta^{C_{1} \epsilon} \nu\left(S^{1}\right)$, and let $E^{\prime}:=\left\{e: I_{s}\left(\pi_{e \sharp} \mu_{B}\right) \leq \delta^{-C_{2}^{\prime} \epsilon}\right\}$. One evidently needs $\geq\left(1-\delta^{C_{1} \epsilon}\right)\left|E_{B}\right|$ arcs of the form $B\left(e, \delta^{1 / 2}\right) \cap S^{1}, e \in E_{B}$ to cover $E^{\prime}$, and this gives rise to a subset $E \subset E_{B}$ with $|E| \geq\left(1-\delta^{C_{1} \epsilon}\right)\left|E_{B}\right|$. I claim that these are of desired "good" vectors.

For every $e \in E$, by definition, there exists a vector $e^{\prime} \in B\left(e, \delta^{1 / 2}\right) \cap S^{1}$ with

$$
\iint \frac{d \mu x d \mu y}{\left|\pi_{e^{\prime}}(x)-\pi_{e^{\prime}}(y)\right|^{s}}=I_{s}\left(\pi_{e^{\prime} \sharp} \mu\right) \lesssim \delta^{-C_{2}^{\prime} \epsilon} .
$$


Now, if $P_{B}^{\prime} \subset P$ is a subset of cardinality $\left|P_{B}^{\prime}\right| \geq \delta^{C_{0} \epsilon}\left|P_{B}\right|$ (as in the statement of the lemma), then the probability measure $\mu^{\prime}$, defined in the obvious way by restricting and re-normalising $\mu_{B}$ to the subset $\delta^{-1 / 2} P_{B}^{\prime}$, still satisfies (4.21) with the $\lesssim$-parameters depending on $C_{0}$. It follows that $\mathcal{H}_{\infty}^{s}\left(\pi_{e^{\prime}}\left(\operatorname{spt} \mu^{\prime}\right)\right) \gtrsim 1$ (with similar dependence on $C_{0}$ ), and hence $\pi_{e^{\prime}}\left(\operatorname{spt} \mu^{\prime}\right)$ contains a $\left(\delta^{1 / 2}, s, C_{2}\right)$-set of cardinality $\geq \delta^{C_{2} \epsilon-s / 2}$ by Proposition 3.5, if $C_{2} \geq 1$ is large enough (depending on $C_{0}$ and $C_{2}^{\prime}$, which just depends on $C_{1}$ ). Since $\pi_{e^{\prime}}\left(\operatorname{spt} \mu^{\prime}\right)$ is contained in the $\delta^{1 / 2}$-neighbourhood of $\pi_{e^{\prime}}\left(\delta^{-1 / 2} P_{B}^{\prime}\right)$, the same conclusion holds for $\pi_{e^{\prime}}\left(\delta^{-1 / 2} P_{B}^{\prime}\right)$. Finally, using $\left|e^{\prime}-e\right| \leq \delta^{1 / 2}$, the conclusion remains valid for $\pi_{e}\left(\delta^{-1 / 2} P_{B}^{\prime}\right)$, and thus, rescaling by $\delta^{1 / 2}$, the projection $\pi_{e}\left(P_{B}^{\prime}\right)$ contains a $(\delta, s)$-set of cardinality $\geq \delta^{C_{2} \epsilon-s / 2}$ for every $e \in E$.

Fix some constants $C_{0}, C_{1} \geq 1$ and let $C_{2}$ be specified by the lemma (the constant $C_{0}$ will be fixed far below, wheres $C_{1}$ will be specified momentarily). I define $\mathcal{T}_{B}^{\prime} \subset \mathcal{T}_{B}^{\prime \prime}$ by selecting the $\geq\left(1-\delta^{C_{1} \epsilon}\right)\left|\mathcal{T}_{B}^{\prime \prime}\right|$ tubes indicated by the lemma. Then, if $C_{1} \geq 1$ is large enough, (4.17) continues to hold for every $q \in P_{B}$, and with $\mathcal{T}_{B}^{\prime \prime}$ replaced by $\mathcal{T}_{B}^{\prime}$ :

$$
\left|\mathcal{T}_{B}^{\prime} \cap \mathcal{T}_{\delta^{1 / 2}}^{B}(p)\right| \geq\left|\mathcal{T}_{B}^{\prime \prime} \cap \mathcal{T}_{\delta^{1 / 2}}(p)\right|-\delta^{C_{1} \epsilon}\left|\mathcal{T}_{B}^{\prime \prime}\right| \approx \delta^{-s / 2}, \quad p \in P_{B}
$$

Note that the parameters in the " $\approx$ "-notation here do not depend on $C_{1}$, assuming that $C_{1}$ is large enough. This completes the first refinement of $\mathcal{T}_{B}^{\prime \prime}$ : roughly speaking, the conclusion was that "without loss of generality", the sets $P_{B}, B \in \mathcal{B}$, can be assumed to have large projections in every direction perpendicular to the tubes meeting $B$. The second refinement (from $\mathcal{T}_{B}^{\prime}$ to $\mathcal{T}_{B}$ ) is concerned with the distribution of the balls $B \in \mathcal{B}$ meeting a fixed $\delta^{1 / 2}$-tube $T \in \mathcal{T}_{\delta^{1 / 2}}$. Roughly speaking, I claim that "without loss of generality", every tube $T \in \mathcal{T}_{\delta^{1 / 2}}$ only meets a $(1-s)$-dimensional family of balls $B \in \mathcal{B}$.

To formalise such thoughts, write

$$
P_{\delta^{1 / 2}}:=\left\{p_{B}: B \in \mathcal{B}\right\} .
$$

Since the balls $B \in \mathcal{B}$ were assumed $\delta^{1 / 2}$-separated (see (4.10)), it follows that $P_{\delta^{1 / 2}}$ is a $\left(\delta^{1 / 2}, 1\right)$-set of cardinality $\left|P_{\delta^{1 / 2}}\right|=|\mathcal{B}| \approx \delta^{-1 / 2}$. Consider the following inequality (see explanations below):

$$
\sum_{T \in \mathcal{T}_{\delta^{1 / 2}}} \sum_{\substack{B, B^{\prime} \in \mathcal{B} \\ B \neq B^{\prime}}} \frac{\chi_{\mathcal{T}_{B}^{\prime} \cap \mathcal{T}_{B^{\prime}}^{\prime}}(T)}{\left|p_{B}-p_{B^{\prime}}\right|^{1-s}}=\sum_{\substack{B, B^{\prime} \in \mathcal{B} \\ B \neq B^{\prime}}} \frac{\left|\mathcal{T}_{B}^{\prime} \cap \mathcal{T}_{B^{\prime}}^{\prime}\right|}{\left|p_{B}-p_{B^{\prime}}\right|^{1-s}} \lesssim \sum_{\substack{B, B^{\prime} \in \mathcal{B} \\ B \neq B^{\prime}}} \frac{1}{\left|p_{B}-p_{B^{\prime}}\right|} \lesssim \delta^{-1} .
$$

The first " $\lesssim$ "-inequality uses the fact that $\mathcal{T}_{B}^{\prime}$ (or $\left.\mathcal{T}_{B^{\prime}}^{\prime}\right)$ is a $\left(\delta^{1 / 2}, s\right)$-set of tubes: since $T \in$ $\mathcal{T}_{B}^{\prime} \cap \mathcal{T}_{B^{\prime}}^{\prime}$ implies that $p_{B}, p_{B^{\prime}} \in T$ (recall (4.18)), this can only hold for $\lesssim 1 /\left|p_{B}-p_{B^{\prime}}\right|^{s}$ choices of $T \in \mathcal{T}_{B}^{\prime}$ (or $T \in \mathcal{T}_{B^{\prime}}^{\prime}$ ). The second "ミ"-inequality in (4.24) follows simply from the fact that $P_{\delta^{1 / 2}}$ is a $\left(\delta^{1 / 2}, 1\right)$-set.

Fix a large constant $C_{3} \geq 1$. It follows from (4.24) and Chebyshev's inequality that

$$
\sum_{\substack{B, B^{\prime} \in \mathcal{B} \\ B \neq B^{\prime}}} \frac{\chi_{\mathcal{T}_{B}^{\prime} \cap \mathcal{T}_{B^{\prime}}^{\prime}}(T)}{\left|p_{B}-p_{B^{\prime}}\right|^{1-s}} \geq \delta^{-C_{3} \epsilon+s-1}
$$

can only hold for $\lesssim \delta^{C_{3} \epsilon-s}$ tubes $T \in \mathcal{T}_{\delta^{1 / 2}}$. Recalling that $\left|\mathcal{T}_{\delta^{1 / 2}}\right| \approx \delta^{-s}$ by (4.14), this roughly says that the tubes satisfying (4.25) are exceptional. I need a the following slightly more accurate statement: for half of the balls $B \in \mathcal{B}$, only a tiny fraction of 
the tubes in $T \in \mathcal{T}_{B}$ can satisfy (4.25), if $C_{3} \geq 1$ was chosen large enough. Indeed, recall the constant $C_{1}$ from the previous page and assume that, for a certain $C_{3} \geq 1$, it holds that $\geq \delta^{C_{1} \epsilon}\left|\mathcal{T}_{B}^{\prime}\right| \approx \delta^{C_{1} \epsilon-s / 2}$ tubes in $\mathcal{T}_{B}^{\prime}$ satisfy (4.25) for $B \in \mathcal{B}^{\prime} \subset \mathcal{B}$, where $\left|\mathcal{B}^{\prime}\right| \geq|\mathcal{B}| / 2$. Then Lemma 4.1 applies at scale $\delta^{1 / 2}$, and with the $\left(\delta^{1 / 2}, 1\right)$-set $\mathcal{B}^{\prime}$, and implies that the total number of tubes in $\mathcal{T}_{\delta^{1 / 2}}$ satisfying (4.25) is $\gtrsim \delta^{-s}$, where the implicit parameters depend on $C_{1}$, but clearly not on $C_{3}$. Comparing this with the upper bound $\lesssim \delta^{C_{3} \epsilon-s}$ gives an upper bound for $C_{3}$, which depends on $C_{1}$.

Hence, assuming that $C_{3} \geq 1$ is large enough, the converse of (4.25) holds for all $B \in \mathcal{B}^{\prime}$, and for $\geq\left(1-\delta^{C_{1} \epsilon}\right)\left|\mathcal{T}_{B}^{\prime}\right|$ tubes in $\mathcal{T}_{B}^{\prime}$. These tubes will be denoted by $\mathcal{T}_{B}$. And once more, if $C_{1} \geq 1$ is large enough, the analogue of (4.22) continues to hold for $\mathcal{T}_{B}$ :

$$
\left|\mathcal{T}_{B} \cap \mathcal{T}_{\delta^{1 / 2}}^{B}(p)\right| \approx \delta^{-s / 2}, \quad p \in P_{B}, B \in \mathcal{B}^{\prime} .
$$

Again, the parameters in the " $\approx$ "-notation do not, in fact, depend on $C_{1}$, assuming that $C_{1}$ is large enough. For each tube $T \in \mathcal{T}_{B}$, I further observe that the number $M_{T}:=\mid\{B \in$ $\left.\mathcal{B}^{\prime}: T \in \mathcal{T}_{B}\right\} \mid$ satisfies

$$
M_{T} \lesssim \delta^{(s-1) / 2}
$$

where the implicit constants depend on $C_{3}$. Indeed, the failure of (4.27) (say: $M_{T} \geq$ $\delta^{-\left(2 C_{3} \epsilon+s-1\right) / 2}$ ) would imply that there are far more than $\delta^{s-1}$ pairs $B, B^{\prime} \in \mathcal{B}^{\prime} \subset \mathcal{B}$ such that $T \in \mathcal{T}_{B} \cap \mathcal{T}_{B^{\prime}} \subset \mathcal{T}_{B}^{\prime} \cap \mathcal{T}_{B^{\prime}}^{\prime}$, which would violate (4.25) (since $\left|p_{B}-p_{B^{\prime}}\right| \lesssim 1$ for all pairs $\left.B, B^{\prime} \in \mathcal{B}\right)$.

4.4. Considerations at scale $\delta$. From now on, only the points in $P_{B}, B \in \mathcal{B}^{\prime}$, play any role in the proof. Set

$$
P^{\prime}:=\bigcup_{B \in \mathcal{B}^{\prime}} P_{B}
$$

which is a $(\delta, 1)$-set of cardinality $\approx \delta^{-1}$, since $\left|\mathcal{B}^{\prime}\right| \approx \delta^{-1 / 2}$ and $\left|P_{B}\right| \approx \delta^{-1 / 2}$ for $B \in \mathcal{B}$ by the definition of $P_{B}$ (just above (4.16)). Fix $p \in P^{\prime}$ and $B \in \mathcal{B}^{\prime}$ such that $p \in P_{B}$. For every

$$
T \in \mathcal{T}_{B} \cap \mathcal{T}_{\delta^{1 / 2}}^{B}(p),
$$

define $\mathcal{T}_{p}^{\prime}$ to consist of all the $\delta$-tubes $\mathcal{T}_{p}$, which are $\delta$-children of $T$. By definition of $\mathcal{T}_{\delta^{1 / 2}}^{B}(p)$ (see (4.15)) and (4.26)), the resulting subset $\mathcal{T}_{p}^{\prime} \subset \mathcal{T}_{p}$ is a $(\delta, s)$-set of tubes containing $p$, and with

$$
\left|\mathcal{T}_{p}^{\prime}\right| \approx \delta^{-s}
$$

Thus, the set $P^{\prime} \subset P$ and the families $\mathcal{T}_{p}^{\prime} \subset \mathcal{T}_{p}, p \in P^{\prime}$, satisfy precisely the same hypotheses as the original families $P$ and $\mathcal{T}_{p}$ in Theorem 4.3. So, for notational, convenience, I re-define $P:=P^{\prime}, \mathcal{T}_{p}:=\mathcal{T}_{p}^{\prime}$, and $\mathcal{B}:=\mathcal{B}^{\prime}$. As before (in Remark 4.6), I continue to assume, without loss of generality, that

$$
\mathcal{T}:=\bigcup_{p \in P} \mathcal{T}_{p}
$$

I also re-define $\mathcal{T}_{\delta^{1 / 2}}$ to be the union of the families $\mathcal{T}_{B}, B \in \mathcal{B}$. Note that, with this definition of $\mathcal{T}_{\delta^{1 / 2}}$, one has (4.27) for all tubes in $\mathcal{T}_{\delta^{1 / 2}}$.

Compared with the original families $\mathcal{T}_{p}$, the new families $\mathcal{T}_{p}$ now enjoy additional regularity properties, which will be useful during the remainder of the proof. To exploit these, I record the following observation: 
Lemma 4.29. Assume that $p \in B \in \mathcal{B}$ and $T \in \mathcal{T}_{p}$. Then the $\delta^{1 / 2}$-parent of $T$ belongs to $\mathcal{T}_{B}$. In particular, the $\delta^{1 / 2}$-parents of the tubes in $\mathcal{T}$ belong to $\mathcal{T}_{\delta^{1 / 2}}$.

Proof. This follows immediately from the construction of $\mathcal{T}_{p}^{\prime}$ - which is now called $\mathcal{T}_{p}$.

For $T \in \mathcal{T}$, write

$$
N_{T}:=\left|\left\{p \in P: T \in \mathcal{T}_{p}\right\}\right|,
$$

which is the analogue of the number $M_{T}$ at scale $\delta$. I make the following (rather familiar) claim: for at least half of the points $p \in P$, only a tiny fraction of the tubes in $\mathcal{T}_{p}$ can fail to satisfy $N_{T} \lesssim \delta^{s-1}$. The proof is virtually the same as for the numbers $M_{T}$. One starts with the inequality

$$
\sum_{\substack{T \in \mathcal{T} \\ p, q \in P \\ p \neq q}} \frac{\chi_{\mathcal{T}_{p} \cap \mathcal{T}_{q}}(T)}{|p-q|^{1-s}} \lesssim \delta^{-2},
$$

which is analogous to and proven in the same way as (4.24). Thus, only $\lesssim \delta^{C_{4} \epsilon-2 s}$ tubes in $\mathcal{T}_{p}$ can satisfy

$$
\sum_{\substack{p, q \in P \\ p \neq q}} \frac{\chi_{\mathcal{T}_{p} \cap \mathcal{T}_{q}}(T)}{|p-q|^{1-s}} \geq \delta^{-C_{4} \epsilon+2 s-2} .
$$

Hence, using Lemma 4.1 as before, the inequality above can only hold for a tiny fraction (depending on $C_{4}$ ) of the tubes in $\mathcal{T}_{p}$, for half of the points in $P$. This implies the statement about the numbers $N_{T}$. Now, as final refinement, I only keep the "good" half of the points in $P$, and for those $p \in P$, I re-define $\mathcal{T}_{p}$ to consist of the tubes $T$ with

$$
N_{T} \lesssim \delta^{s-1} \text {. }
$$

If $C_{4}$ was large enough, the cardinality estimate (4.28) stays valid. Finally, if $\mathcal{T}$ is redefined as the union of the (remaining) tubes in $\mathcal{T}_{p}, p \in P$, one may assume that (4.30) holds uniformly for all $T \in \mathcal{T}$.

Fix $p \in P$ and $T \in \mathcal{T}$. Recall that the pair $(p, T)$ is called an incidence, if $T \in \mathcal{T}_{p}$, and the collection of all incidences is denoted by $I(P, \mathcal{T}):=\left\{(p, T): T \in \mathcal{T}_{p}\right\}$. Evidently

$$
|I(P, \mathcal{T})|=\sum_{p \in P}\left|\mathcal{T}_{p}\right| \approx \delta^{-s-1}
$$

By the uniform upper bound (4.30), any tube $T \in \mathcal{T}$ can only be incident to $\lesssim \delta^{s-1}$ points in $P$. Since $|\mathcal{T}| \lesssim \delta^{-2 s}$ by the main counter assumption (4.8), the estimate (4.31) shows that there exist $\approx \delta^{-2 s}$ tubes in $\mathcal{T}$ with $N_{T} \approx \delta^{s-1}$. These tubes will be called good tubes, and they will be denoted by $\mathcal{T}^{G}$.

Lemma 4.32. Any fixed tube $T_{0} \in \mathcal{T}_{\delta^{1 / 2}}$ can only have $\lesssim \delta^{-s}$ children in $\mathcal{T}^{G}$.

Proof. Write $\mathcal{T}^{G}\left(T_{0}\right):=\left\{T \in \mathcal{T}^{G}: T \subset T_{0}\right\}$, and let $I^{G}\left(T_{0}\right)$ be the set of incidences

$$
I^{G}\left(T_{0}\right):=\left\{(p, T) \in P \times \mathcal{T}: T \in \mathcal{T}_{p} \cap \mathcal{T}^{G}\left(T_{0}\right)\right\} .
$$

By the definition of good tubes, evidently

$$
\left|I^{G}\left(T_{0}\right)\right| \gtrsim\left|\mathcal{T}^{G}\left(T_{0}\right)\right| \delta^{s-1} .
$$


On the other hand, by Lemma 4.29, an incidence $(p, T) \in P \times \mathcal{T}_{p}$ can only occur, if the $\delta^{1 / 2}$-parent of $T$ belongs to $\mathcal{T}_{B_{p}}$ for the (unique) ball $B_{p} \in \mathcal{B}$ containing $p$. For $T \subset T_{0}$, the $\delta^{1 / 2}$-parent is evidently $T_{0}$, so

$$
T \subset T_{0} \text { and }(p, T) \in \mathcal{T}_{p} \quad \Longrightarrow \quad T_{0} \in \mathcal{T}_{B_{p}} .
$$

Now, recall from the estimate $M_{T_{0}} \lesssim \delta^{(s-1) / 2}$ (see (4.27)), that there are only $\lesssim \delta^{(s-1) / 2}$ balls $B$ with $T_{0} \in \mathcal{T}_{B}$. For every such a ball $B$, every point $p \in P_{B}$ can be incident to $\lesssim \delta^{-s / 2}$ tubes $T \in \mathcal{T}_{p}$ with $T \subset T_{0}$ (for the simple reason that $T_{0}$ only contains $\lesssim \delta^{-s / 2}$ tubes in $\mathcal{T}_{p}$ by Lemma 3.16). Recalling that $\left|P_{B}\right| \lesssim \delta^{-1 / 2}$, this gives the upper bound

$$
\left|I^{G}\left(T_{0}\right)\right| \leq \sum_{B: T_{0} \in \mathcal{T}_{B}}\left|\left\{(p, T) \in B \times \mathcal{T}: T \in \mathcal{T}_{p} \cap \mathcal{T}^{G}\left(T_{0}\right)\right\}\right| \lesssim \delta^{(s-1) / 2} \cdot \delta^{-1 / 2} \cdot \delta^{-s / 2}=\delta^{-1} .
$$

Comparing with the lower bound for $\left|I^{G}\left(T_{0}\right)\right|$ completes the proof.

To sum up the most recent observations, there are $\approx \delta^{-2 s}$ good tubes, each one of which is contained in some tube of $\mathcal{T}_{\delta^{1 / 2}}$, and each tube in $\mathcal{T}_{\delta^{1 / 2}}$ can only contain $\lesssim \delta^{-s}$ good tubes. By the main counter assumption (4.8), moreover, one has $\left|\mathcal{T}_{\delta^{1 / 2}}\right| \lesssim \delta^{-s}$, which finally implies that there exists a tube $T_{0} \in \mathcal{T}$ with $\left|\mathcal{T}^{G}\left(T_{0}\right)\right| \approx \delta^{-s}$. For simplicity, write

$$
\mathcal{T}_{0}:=\mathcal{T}^{G}\left(T_{0}\right) .
$$

I now claim that there are $\approx \delta^{(s-1) / 2}$ balls $B \in \mathcal{B}$, say $\mathcal{B}_{0}$, such that $T_{0} \in \mathcal{T}_{B}$ for $B \in \mathcal{B}_{0}$, and such that in each ball $B \in \mathcal{B}_{0}$ one finds $\approx \delta^{-1 / 2}$ points of $P_{B}$, say $P_{B}^{\prime}$, with a nearmaximal number of incidences with $\mathcal{T}_{0}$, namely

$$
\left|\mathcal{T}_{p} \cap \mathcal{T}_{0}\right| \approx \delta^{-s / 2}, \quad p \in P_{B}^{\prime}, B \in \mathcal{B}_{0} .
$$

This follows directly from the proof of Lemma 4.32. First observe that $\left|I^{G}\left(T_{0}\right)\right| \approx \delta^{-1}$, since $\left|\mathcal{T}_{0}\right| \approx \delta^{-s}$. Next, have a look at the upper bound (4.33), and observe that if any part of the claim failed, the bound would be lower than $\delta^{-1}$. This establishes the claim.

Now, since $T_{0} \in \mathcal{T}_{\delta^{1 / 2}}$, the converse of (4.25) holds for $T_{0}$ (recall the definition of $\mathcal{T}_{B}$ next to (4.26), and recall that every tube in $\mathcal{T}_{\delta^{1 / 2}}$ belongs to $\mathcal{T}_{B}$ for some $B$ ):

$$
\sum_{\substack{B, B \in \mathcal{B}_{0} \\ B \neq B^{\prime}}} \frac{1}{\left|p_{B}-p_{B^{\prime}}\right|^{1-s}} \lesssim \delta^{s-1} .
$$

Using Chebyshev's inequality, this implies that a further subset $\mathcal{B}_{0}^{\prime} \subset \mathcal{B}_{0}$ of cardinality $\left|\mathcal{B}_{0}^{\prime}\right| \approx \delta^{(s-1) / 2}$ satisfies

$$
\sum_{B^{\prime} \in \mathcal{B}_{0}} \frac{1}{\left|p_{B}-p_{B}^{\prime}\right|^{1-s}} \lesssim \delta^{(s-1) / 2}, \quad B \in \mathcal{B}_{0}^{\prime} .
$$

and then $\mathcal{B}_{0}^{\prime}$ is a $\left(\delta^{1 / 2}, 1-s\right)$-set of cardinality $\approx \delta^{(s-1) / 2}$. Since the balls $\mathcal{B}_{0}^{\prime}$ satisfy precisely the same estimates as $\mathcal{B}_{0}$, I will continue writing $\mathcal{B}_{0}:=\mathcal{B}_{0}^{\prime}$. For convenience, assume that $T_{0}$ is a vertical tube (that is, change coordinates so that this holds). Then the $y$-coordinates of the points $p_{B} \in B, B \in \mathcal{B}_{0}$, form a $\left(\delta^{1 / 2}, 1-s\right)$-set in $[-1,1]$. Denote these $y$-coordinates by $A_{1}:=\left\{p_{B}^{y}, B \in \mathcal{B}_{0}\right\}$. 
4.5. Quasi-product sets, and concluding the proof of Theorem 4.3. Now, recall (from above (4.35)) the subsets $P_{B}^{\prime} \subset P_{B}$, defined for $B \in \mathcal{B}_{0}$. They have cardinality $\left|P_{B}^{\prime}\right| \geq$ $\delta^{C_{0} \epsilon}\left|P_{B}\right|$ for some constant $C_{0} \geq 1$. This is the constant with which one wants to apply Lemma 4.19: since $T_{0} \in \mathcal{T}_{B}^{\prime}$ (recall (4.22)), the projection of $\pi\left(P_{B}^{\prime}\right)$ of $P_{B}^{\prime}$ to the $x$-axis contains a $(\delta, s)$-set $\Delta_{B}$ of cardinality $\left|\Delta_{B}\right| \geq \delta^{C_{2} \epsilon-s / 2}$.

Consider the "quasi-product set"

$$
F^{\prime}:=\bigcup_{p_{B}^{y} \in A_{1}} \Delta_{B} \times\left\{p_{B}^{y}\right\}
$$

Fix $(a, b)=\left(a, p_{B}^{y}\right) \in F^{\prime}, B \in \mathcal{B}_{0}$. Then $a=\pi(p)$ for some $p=p_{(a, b)} \in P_{B}^{\prime}$, so that $|(a, b)-p| \leq 2 \delta^{1 / 2}$. Recall that (4.35) holds for $p$, and let $T \in \mathcal{T}_{p} \cap \mathcal{T}_{0}$. By elementary geometry, using $|(a, b)-p| \leq 2 \delta^{1 / 2}$ and $p \in T \subset T_{0}$, the point $(a, b) \in F^{\prime}$ is covered by $B(0,10) \cap T^{\prime}$ for some dyadic $\delta$-tube $T^{\prime}$ in the $C \delta$-neighbourhood of $T$. (By this, I mean that if $T=\mathcal{D}(Q)$, then $T^{\prime}=\mathcal{D}\left(Q^{\prime}\right)$ for some dyadic $\delta$-square $Q^{\prime}$ with $\operatorname{dist}\left(Q, Q^{\prime}\right) \leq C \delta$.) This is best explained by a picture, see Figure 3 .

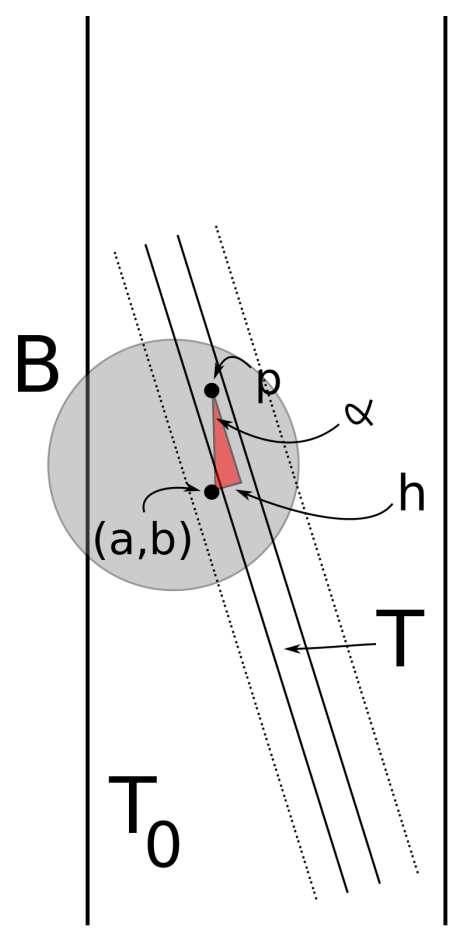

FIGURE 3. The geometry of the tubes $T_{0}, T$ and the points $(a, b)$ and $p=$ $p_{(a, b)}$. Since $T \subset T_{0}$, the angle $\alpha$ is $\lesssim \delta^{1 / 2}$. Since $p,(a, b) \in B$, the distance between the points $p$ and $(a, b)$ is no greater than $2 \delta^{1 / 2}$. Hence $h \lesssim \delta$, which ensures that $(a, b)$ is contained in $B(0,10) \cap T^{\prime}$ for some dyadic $\delta$-tube $T^{\prime}$ parallel to, and at distance $\lesssim \delta$, from $T \in \mathcal{T}_{p}$. 
Now, for each $T \in \mathcal{T}_{0}$, choose an ordinary $C \delta$-tube parallel to $T$, which covers $B(0,10) \cap$ $T^{\prime}$ for all the dyadic $\delta$-tubes $T^{\prime}$ in the $C \delta$-neighbourhood of $T$. The collection of all ordinary $C \delta$-tubes so obtained is denoted by $\mathcal{T}^{o}$ (here " $o$ " stands for "ordinary"). Then

$$
\left|\mathcal{T}^{o}\right| \sim\left|\mathcal{T}_{0}\right| \approx \delta^{-s} \text {. }
$$

In particular, $\mathcal{T}^{o}$ contains the ordinary $C \delta$-tubes produced from the dyadic $\delta$-tubes in $\mathcal{T}_{p_{(a, b)}} \cap \mathcal{T}_{0}$. By (4.35) and the discussion above, this gives rise to a $(\delta, s)$-subset $\mathcal{T}_{(a, b)}^{o} \subset \mathcal{T}^{o}$ of ordinary $C \delta$-tubes of cardinality $\left|\mathcal{T}_{(a, b)}^{o}\right| \approx \delta^{-s / 2}$, with the property that

$$
(a, b) \in T, \quad T \in \mathcal{T}_{(a, b)}^{o} .
$$

Finally, consider the following affine transformation of $F^{\prime}$ :

$$
F:=\bigcup_{b \in A_{1}} A_{b} \times\{b\}
$$

where $A_{b}:=\delta^{-1 / 2} \Delta_{B}$. Note that each $A_{b}$ is a $\left(\delta^{1 / 2}, s\right)$-set, and recall that $A_{1}$ is a $\left(\delta^{1 / 2}, 1-\right.$ $s)$-set. Clearly $F=\operatorname{Aff}\left(F^{\prime}\right)$, where $\operatorname{Aff}(x, y)=\left(\delta^{-1 / 2} x, y\right)$. Then $\mathcal{T}^{\prime}:=\operatorname{Aff}\left(\mathcal{T}^{o}\right)=$ $\left\{\operatorname{Aff}(T): T \in \mathcal{T}^{o}\right\}$ is a family of ordinary $C^{\prime} \delta^{1 / 2}$-tubes of cardinality $\left|\mathcal{T}^{\prime}\right| \sim|\mathcal{T}| \approx \delta^{-s}$. Moreover, every point $x=\left(\delta^{-1 / 2} a, b\right) \in F$ is is contained in a $\left(\delta^{1 / 2}, s\right)$-subset $\mathcal{T}_{x}^{\prime} \subset \mathcal{T}^{\prime}$ of ordinary $C^{\prime} \delta^{1 / 2}$-tubes with $\left|\mathcal{T}_{x}^{\prime}\right| \approx \delta^{-s / 2}$, namely $\mathcal{T}_{x}^{\prime}:=\operatorname{Aff}\left(\mathcal{T}_{(a, b)}^{o}\right)$. The existence of $F$ and the families $\mathcal{T}^{\prime}, \mathcal{T}_{x}^{\prime}$, now contradict the next proposition (at scale $\delta^{1 / 2}$, with $P=F$, $\mathcal{T}=\mathcal{T}^{\prime}$ and $\tau=1-s>0$ ). This completes the proof of Theorem 4.3.

4.6. An incidence theorem for quasi-product sets. The wording "quasi-product set" is rather informal, and simply refers to sets $P$ of the form (4.37) below (if all the sets $A_{b}$ were the same, then $P$ would truly be a product set). On the last meters of the proof above, such a set, namely $F$, was constructed: it turned out that the points $x \in F$ were each incident to a large family $\mathcal{T}_{x}^{\prime}$ of not-too-concentrated tubes, and all the families $\mathcal{T}_{x}^{\prime}$ were subsets of a fixed small family $\mathcal{T}^{\prime}$. The next, and final, proposition shows that this is simply not possible.

Proposition 4.36. Given $0<s<1$ and $\tau>0$, there exists a number $\epsilon=\epsilon(s, \tau)>0$ such that the following holds. Let $B \subset[0,1]$ be a $\left(\delta, \tau, \delta^{-\epsilon}\right)$-set of cardinality $|B| \gtrsim \delta^{-\tau+\epsilon}$, and for each $b \in B$, assume that $A_{b} \subset[0,1]$ is a $\left(\delta, s, \delta^{-\epsilon}\right)$-set of cardinality $\left|A_{b}\right| \gtrsim \delta^{-s+\epsilon}$. Consider the $\left(\delta, s+\tau, \delta^{-2 \epsilon}\right)$-set

$$
P:=\bigcup_{b \in B} A_{b} \times\{b\}
$$

Assume that $\mathcal{T}$ is a collection of (ordinary) $\delta$-tubes such every family $\{T \in \mathcal{T}: p \in T\}, p \in P$, contains in $a\left(\delta, s, \delta^{-\epsilon}\right)$-set $\mathcal{T}_{p} \subset \mathcal{T}$ with $\left|\mathcal{T}_{p}\right| \gtrsim \delta^{-s+\epsilon}$. Then $|\mathcal{T}| \gtrsim \delta^{-2 s-\epsilon}$.

The proof of Proposition 4.36 is, again, based on a counter assumption, namely $|\mathcal{T}| \leq$ $\delta^{-2 s-\epsilon}$. For the remainder of the paper, the notations $\lesssim, \gtrsim$ and $\approx$, and the concept of $(\delta, t)$ set, are defined exactly as before, in Section 4.1 , but now relative to the " $\epsilon$ " in this counter assumption. Naturally, the implicit constants $C, C_{\epsilon}$ are now also allowed to depend on $\tau$, in addition to $s$.

Before starting the proof of Proposition 4.36 in earnest, I recall two standard results from additive combinatorics. The first is the Balog-Szemerédi-Gowers theorem. The statement below is taken verbatim from p. 196 in [2]. For a proof, see [21], p. 267. 
Theorem 4.38 (Balog-Szemerédi-Gowers). There exists an absolute constant $C \geq 1$ such that the following holds. Let $A, B \subset \mathbb{R}$ be finite sets, and assume that $G \subset A \times B$ is a set of pairs such that

$$
|G| \geq \frac{|A||B|}{K} \text { and }|\{x+y:(x, y) \in G\}| \leq K|A|^{1 / 2}|B|^{1 / 2}
$$

for some $K>1$. Then, there exist $A^{\prime} \subset A$ and $B^{\prime} \subset B$ satisfying

- $\left|A^{\prime}\right| \geq K^{-C}|A|,\left|B^{\prime}\right| \geq K^{-C}|B|$,

- $\left|A^{\prime}+B^{\prime}\right| \leq K^{C}|A|^{1 / 2}|B|^{1 / 2}$, and

- $\left|G \cap\left(A^{\prime} \times B^{\prime}\right)\right| \geq K^{-C}|A||B|$.

The second auxiliary result is the Plünnecke-Ruzsa inequality, whose proof can also be found in [21]:

Theorem 4.39 (Plünnecke-Ruzsa). Assume that $A, B \subset \mathbb{R}$ are finite sets such that

$$
|A+B| \leq C|A|
$$

for some integer $C \geq 1$. Then

for all $m, n \in \mathbb{N}$.

$$
\left|B^{m} \pm B^{n}\right| \leq C^{m+n}|A|
$$

Remark 4.40. Theorem 4.39 will be applied in the following form: if $A, B \subset \mathbb{R}$ are $\delta$ separated sets with $|A| \approx|B|$ and

$$
N(A+B, \delta) \lesssim|A|,
$$

then $N(B+B, \delta) \lesssim|A|$. This statement follows easily from Theorem 4.39 by considering the sets $[A]_{\delta}=\left\{[a]_{\delta}: a \in A\right\} \subset \delta \mathbb{Z}$ and $[B]_{\delta}:=\left\{[b]_{\delta} ; b \in B\right\} \subset \delta \mathbb{Z}$, where $[x]_{\delta} \in \delta \mathbb{Z}$ stands for the largest number $\delta n \in \delta \mathbb{Z}$ satisfying $\delta n \leq x$. Then the hypothesis $N(A+B, \delta) \lesssim|A|$ implies that $\left|[A]_{\delta}+[B]_{\delta}\right| \lesssim|[A]|_{\delta}$, so Theorem 4.39 can be applied.

Proof of Proposition 4.36. I start by making three convenient extra assumptions, which are not difficult to arrange. First, every tube in $\mathcal{T}$ meets only one point in each set $A_{b} \times\{b\}$ (that is, the tubes in $\mathcal{T}$ are "roughly vertical"); this can be arranged by restricting attention to those tubes in each $\mathcal{T}_{p}$, which form an angle $\gtrsim 1$ with horizontal lines. By the $(\delta, s)$-set hypothesis, $\approx \delta^{-s}$ tubes remain in each $\mathcal{T}_{p}$, and then one can re-define $\mathcal{T}$ as the union of the reduced families $\mathcal{T}_{p}$. In particular, now each tube in $\mathcal{T}$ only intersects the lines $\mathbb{R} \times\{b\}, b \in B$, inside a single interval of length $\lesssim \delta$. After this procedure, one can remove some points from each $A_{b} \times\{b\}$ so that the mutual separation exceeds the length of the intervals mentioned above; again, by the $(\delta, s)$-set hypothesis, this can be arranged to that $\approx \delta^{-s}$ points remain for every $b$.

Second,

$$
A_{b} \subset \delta \mathbb{Z}, \quad b \in B .
$$

This can be arranged by perturbing the points of $A_{b}$ by $\leq \delta$. The tubes in $\mathcal{T}_{p}$ may no longer contain $p$, but the $100 \delta$-neighbourhoods of the tubes in $\mathcal{T}_{p}$ certainly do. These neighbourhoods can be covered by 200 ordinary $\delta$-tubes, each, which gives rise to a new family $\mathcal{T}^{\prime}$ of ordinary $\delta$-tubes with $\left|\mathcal{T}^{\prime}\right| \sim|\mathcal{T}|$. Then, one can prove the proposition for $\mathcal{T}^{\prime}$ instead of $\mathcal{T}$.

Third, if $b_{1}, b_{2}, b_{3} \in B$, and $T, T^{\prime} \in \mathcal{T}$ are two tubes both containing certain points $\left(a_{1}, b_{1}\right) \in A_{b_{1}} \times\left\{b_{1}\right\}$ and $\left(a_{3}, b_{3}\right) \in A_{b_{3}} \times\left\{b_{3}\right\}$, then $T \cup T^{\prime}$ can only contain one point in $A_{b_{2}} \times\left\{b_{2}\right\}$. This is similar to the first reduction: it follows from the assumption $T \cup T^{\prime}$ 
intersects $\mathbb{R} \times\left\{b_{2}\right\}$ inside a single interval of length $\lesssim \delta$ (since $T \cup T^{\prime}$ is contained in the $\delta$-neighbourhood of the line connecting $\left(a_{1}, b_{1}\right)$ to $\left(a_{3}, b_{3}\right)$, and both tubes were already assumed to be roughly vertical). Thus, if the separation of $A_{b_{2}}$ exceeds the length of any such interval, the claim is true. And this can, as before, be arranged by discarding a few points from $A_{2}$.

The proof starts in earnest now, and I make the counter assumption $|\mathcal{T}| \leq \delta^{-2 s-\epsilon}$, or, in short,

For $T \in \mathcal{T}$, write

$$
|\mathcal{T}| \lesssim \delta^{-2 s}
$$

$$
N_{T}:=\left|\left\{p \in P: T \in \mathcal{T}_{p}\right\}\right| .
$$

Then, by the first "convenient extra assumption" above, one has the uniform bound

$$
N_{T} \leq|B| \lesssim \delta^{-\tau}
$$

On the other hand

$$
\sum_{T \in \mathcal{T}} N_{T}=\sum_{p \in P}\left|\mathcal{T}_{p}\right| \gtrsim|P| \delta^{-s}=\delta^{-2 s-\tau}
$$

By the counter assumption (4.41), one sees that $N_{T} \approx \delta^{-\tau}$ for $\approx \delta^{-2 s}$ tubes in $\mathcal{T}$. Consequently,

$$
\sum_{T \in \mathcal{T}}\left|\left\{(p, q) \in P \times P: p \sim_{T} q\right\}\right| \gtrsim \delta^{-2 s-2 \tau},
$$

where $p \sim_{T} q$, if and only if $p \neq q$ and $T \in \mathcal{T}_{p} \cap \mathcal{T}_{q}$. Write $p \sim q$, if $p \sim_{T} q$ for some $T \in \mathcal{T}$, and $Q:=\{(p, q): p \sim q\}$. Then the left hand side of the inequality above can be re-written and estimated as

$$
\sum_{p \sim q}\left|\mathcal{T}_{p} \cap \mathcal{T}_{q}\right| \lesssim \sum_{p \sim q} \frac{1}{|p-q|^{s}} \lesssim|Q|^{1 / r_{1}}\left(\sum_{p \neq q} \frac{1}{|p-q|^{s+\tau}}\right)^{1 / r_{2}} \lesssim|Q|^{1 / r_{1}}|P|^{2 / r_{2}} .
$$

The first inequality follows from the $(\delta, s)$-set hypothesis of either $\mathcal{T}_{p}$ or $\mathcal{T}_{q}$ (as in (4.24)). The numbers $r_{1}, r_{2}>1$ are dual exponents such that $s r_{2}=s+\tau$, and the last inequality follows from the fact that $P$ is a $(\delta, s+\tau)$-set with $|P| \approx \delta^{-s-\tau}$. From this and (4.43), one infers that

$$
|Q| \gtrsim|P|^{2} \text {. }
$$

In heuristic terms, this shows that the graph with vertex set $P$ and edge set $\{(p, q): p \sim q\}$ has almost maximal connectivity. Since there are no "edges" $p \sim q$ with $p, q \in A_{b} \times\{b\}$ for any fixed $b \in B$, the inequality above implies

$$
\sum_{b_{1} \neq b_{2}}\left|\left\{(p, q) \in A^{b_{1}} \times A^{b_{2}}: p \sim q\right\}\right|=|Q| \gtrsim|P|^{2} \approx \delta^{-2 s-2 \tau},
$$

where $A^{b_{i}}=A_{b_{i}} \times\left\{b_{i}\right\}$.

Let $b_{1}, b_{2} \in B$, and assume that $(p, q) \in A^{b_{1}} \times A^{b_{2}}$ satisfy $p \sim q$. Then, by definition, there exists at least one tube $T_{(p, q)} \in \mathcal{T}_{p} \cap \mathcal{T}_{q}$. If there are several, pick exactly one and call it $T_{(p, q)}$. Also, make these choices so that $T_{(p, q)}=T_{(q, p)}$. Then, set

$$
\mathcal{T}^{b_{1}, b_{2}}:=\left\{T_{(p, q)}:(p, q) \in A^{b_{1}} \times A^{b_{2}} \text { and } p \sim q\right\},
$$

and note that

$$
\mathcal{T}^{b_{1}, b_{2}}=\mathcal{T}^{b_{2}, b_{1}}, \quad b_{1}, b_{2} \in B .
$$


Now, if $\left(p_{1}, q_{1}\right),\left(p_{2}, q_{2}\right) \in A^{b_{1}} \times A^{b_{2}}$ are two distinct pairs, then the collections $\mathcal{T}_{p_{1}} \cap \mathcal{T}_{q_{1}}$ and $\mathcal{T}_{p_{2}} \cap \mathcal{T}_{q_{2}}$ are disjoint. Indeed, if $p_{1} \neq p_{2}$, say, then no tube can lie in both $\mathcal{T}_{p_{1}}$ and $\mathcal{T}_{p_{2}}$ (since this would imply $p_{1} \sim p_{2}$ ). This implies that $T_{\left(p_{1}, q_{1}\right)} \neq T_{\left(p_{2}, q_{2}\right)}$, and consequently $\left|\mathcal{T}^{b_{1}, b_{2}}\right| \geq\left|\left\{(p, q) \in A^{b_{1}} \times A^{b_{2}}: p \sim q\right\}\right|$. Hence

$$
\sum_{b_{1}, b_{2}}\left|\mathcal{T}^{b_{1}, b_{2}}\right| \gtrsim|P|^{2} \approx \delta^{-2 s-2 \tau}
$$

by (4.44). Now, using the counter assumption $|\mathcal{T}| \lesssim \delta^{-2 s}$, and recalling (4.45), one can perform the following estimate:

$$
\begin{aligned}
\sum_{b_{1}, b_{2}, b_{3}}\left|\mathcal{T}^{b_{1}, b_{2}} \cap \mathcal{T}^{b_{2}, b_{3}}\right| & =\sum_{T \in \mathcal{T}} \sum_{b_{2}} \sum_{b_{1}, b_{3}} \chi_{\mathcal{T}^{b_{1}, b_{2}}}(T) \chi_{\mathcal{T}^{b_{2}, b_{3}}}(T) \\
& =\sum_{T \in \mathcal{T}} \sum_{b_{2}}\left(\sum_{b} \chi_{\mathcal{T}^{b, b_{2}}}(T)\right)^{2} \\
& \geq \frac{1}{|\mathcal{T}||B|}\left(\sum_{T \in \mathcal{T}} \sum_{b, b_{2}} \chi_{\mathcal{T}^{b, b_{2}}}(T)\right)^{2} \\
& \gtrsim \frac{|P|^{4}}{|\mathcal{T}||B|} \gtrsim \delta^{-2 s}|B|^{3} .
\end{aligned}
$$

Since evidently $\left|\mathcal{T}^{b_{1}, b_{2}} \cap \mathcal{T}^{b_{2}, b_{3}}\right| \leq|\mathcal{T}| \lesssim \delta^{-2 s}$ for any triple $\left(b_{1}, b_{2}, b_{3}\right)$, it follows that there exist $\approx|B|^{3}$ triples $\left(b_{1}, b_{2}, b_{3}\right)$ with the property that

$$
\left|\mathcal{T}^{b_{1}, b_{2}} \cap \mathcal{T}^{b_{2}, b_{3}}\right| \approx \delta^{-2 s}
$$

As will be made precise in a moment, the condition $\left|\mathcal{T}^{b_{1}, b_{2}} \cap \mathcal{T}^{b_{2}, b_{3}}\right| \approx \delta^{-2 s}$ roughly means that there are $\approx \delta^{-2 s}$ points in $A_{b_{1}} \times A_{b_{3}}$ such that the projection of these points is small in a certain direction, determined by $b_{1}, b_{2}, b_{3}$.

Consider a triple of distinct points $b_{1}, b_{2}, b_{3} \in B^{3}$ with $\mathcal{T}^{b_{1}, b_{2}} \cap \mathcal{T}^{b_{2}, b_{3}} \neq \emptyset$. Fix $T \in$ $\mathcal{T}^{b_{1}, b_{2}} \cap \mathcal{T}^{b_{2}, b_{3}}$. Since $T \in \mathcal{T}^{b_{1}, b_{2}}$, one has $T \in \mathcal{T}_{p_{1}} \cap \mathcal{T}_{p_{2}}$ for some unique pair of points

$$
p_{1}=\left(a_{1}, b_{1}\right) \in A^{b_{1}} \quad \text { and } \quad p_{2}=\left(a_{2}, b_{2}\right) \in A^{b_{2}} .
$$

Similarly, because $T \in \mathcal{T}^{b_{2}, b_{3}}$, there exists yet another unique point

$$
p_{3}=\left(a_{3}, b_{3}\right) \in A^{b_{3}}
$$

such that $T \in \mathcal{T}_{p_{2}} \cap \mathcal{T}_{p_{3}}$. In particular, gathering all the pairs $\left(a_{1}, a_{3}\right) \in A_{b_{1}} \times A_{b_{3}}$ obtained this way, one sees that the tubes $T \in \mathcal{T}^{b_{1}, b_{2}} \cap \mathcal{T}^{b_{2}, b_{3}}$ give rise to a subset

$$
G_{b_{1}, b_{2}, b_{3}}^{\prime} \subset A_{b_{1}} \times A_{b_{3}} .
$$

of cardinality

$$
\left|G_{b_{1}, b_{2}, b_{3}}^{\prime}\right|=\left|\mathcal{T}^{b_{1}, b_{2}} \cap \mathcal{T}^{b_{2}, b_{3}}\right| .
$$

To see the cardinality claim, one needs to check that distinct tubes $T, T^{\prime} \in \mathcal{T}^{b_{1}, b_{2}} \cap \mathcal{T}^{b_{2}, b_{3}}$ give rise to distinct pairs in $\left(a_{1}, a_{3}\right),\left(a_{1}^{\prime}, a_{3}^{\prime}\right) \in A_{b_{1}} \times A_{b_{3}}$. For $T$ and $T^{\prime}$, let $p_{1}, p_{2}, p_{3}$ and $p_{1}^{\prime}, p_{2}^{\prime}, p_{3}^{\prime}$ be the unique points above. Suppose, for contradiction, that $a_{1}=a_{1}^{\prime}$ and $a_{3}=a_{3}^{\prime}$, which means that $p_{1}=p_{1}^{\prime}$ and $p_{3}=p_{3}^{\prime}$. Then $T, T^{\prime} \in \mathcal{T}_{p_{1}} \cap \mathcal{T}_{p_{2}}$ and $T, T^{\prime} \in$ $\mathcal{T}_{p_{1}} \cap \mathcal{T}_{p_{2}^{\prime}}$. This implies that $p_{2} \neq p_{2}^{\prime}$, since otherwise two tubes in $T, T^{\prime} \in \mathcal{T}_{p_{1}} \cap \mathcal{T}_{p_{2}}$ would 
have been chosen to $\mathcal{T}^{b_{1}, b_{2}}$ contrary to the construction. But then $T, T^{\prime}$ are tubes both containing the points $p_{1} \in A^{b_{1}}$ and $p_{3} \in A^{b_{3}}$ such that the union $T \cup T^{\prime}$ contains two distinct points $p_{2}, p_{2}^{\prime} \in A^{b_{2}}$. This contradicts the third "convenient extra assumption" made at the beginning of the proof, and establishes (4.48).

From now on, restrict attention to triples $\left(b_{1}, b_{2}, b_{3}\right) \in B^{3}$ such that

$$
\min _{i \neq j}\left|b_{i}-b_{j}\right| \approx 1 \text {. }
$$

Since the set of triples satisfying $\min \left|b_{i}-b_{j}\right| \leq \delta^{C \epsilon}$ for $C \geq 1$ has cardinality no larger than $\lesssim \delta^{C \tau \epsilon-\tau}|B|^{2} \lesssim \delta^{C \tau \epsilon}|B|^{3}$ (using the $(\delta, \tau)$-set hypothesis of $B$ ), a large enough choice of $C$, depending on $\tau$, guarantees that $\left|\mathcal{T}^{b_{1}, b_{2}} \cap \mathcal{T}^{b_{2}, b_{3}}\right| \approx \delta^{-2 s}$ holds for $\approx|B|^{3}$ triples satisfying (4.49). Fix one such triple, and consider a pair $\left(a_{1}, a_{3}\right) \in G_{b_{1}, b_{2}, b_{3}}^{\prime}$. Recall how such points arise, and the notation for $p_{1}, p_{2}, p_{3}$. Let

$$
L=\left\{x=\frac{a_{3}-a_{1}}{b_{3}-b_{1}} y+\frac{a_{1} b_{3}-a_{3} b_{1}}{b_{3}-b_{1}}: y \in \mathbb{R}\right\}
$$

be the line spanned by $p_{1}$ and $p_{3}$; then, since $p_{1}, p_{2}, p_{3}$ all lie in the common $\delta$-tube $T \in \mathcal{T}$, the line $L$ passes at distance $\lesssim \delta$ from $p_{2}=\left(a_{2}, b_{2}\right) \in A_{b_{2}} \times\left\{b_{2}\right\}$, which implies

$$
\left|\frac{a_{3}\left(b_{2}-b_{1}\right)+a_{1}\left(b_{3}-b_{2}\right)}{b_{3}-b_{1}}-a_{2}\right| \lesssim \delta,
$$

using the fact that the tubes in $\mathcal{T}$ are nearly vertical. Recalling (4.49), this further implies that

$$
\left|\left(a_{1}+\frac{b_{2}-b_{1}}{b_{3}-b_{2}} a_{3}\right)-\frac{b_{3}-b_{1}}{b_{3}-b_{2}} a_{2}\right| \lesssim \delta \text {. }
$$

Consequently, if $\pi_{b_{1}, b_{2}, b_{3}}$ stands for the projection-like mapping

$$
\pi_{b_{1}, b_{2}, b_{3}}(x, y)=x+\frac{b_{2}-b_{1}}{b_{3}-b_{2}} y,
$$

then $\pi_{b_{1}, b_{2}, b_{3}}\left(G_{b_{1}, b_{2}, b_{3}}^{\prime}\right)$ is contained in the $\lesssim \delta$-neighbourhood of

$$
\frac{b_{3}-b_{1}}{b_{3}-b_{2}} A_{b_{2}} \text {. }
$$

Observing that $N\left(\left[\left(b_{3}-b_{1}\right) /\left(b_{3}-b_{2}\right)\right] A_{b_{2}}, \delta\right) \lesssim \delta^{-s}$ by (4.49), it follows that

$$
N\left(\pi_{b_{1}, b_{2}, b_{3}}\left(G_{b_{1}, b_{2}, b_{3}}^{\prime}\right), \delta\right) \lesssim \delta^{-s} .
$$

This holds for any triple $\left(b_{1}, b_{2}, b_{3}\right) \in B^{3}$ satisfying (4.49) by definition of $G_{b_{1}, b_{2}, b_{3}}^{\prime}$, but the information is most useful, if $\left|G_{b_{1}, b_{2}, b_{3}}^{\prime}\right| \approx \delta^{-2 s} \approx\left|A_{b_{1}} \times A_{b_{3}}\right|$, which holds for $\approx|B|^{3}$ triples (recall (4.48) and (4.47)). Write

$$
F_{b_{1}, b_{2}, b_{3}}:=\left\{\left(a_{1},\left[\frac{b_{2}-b_{1}}{b_{3}-b_{2}} a_{3}\right]_{\delta}\right):\left(a_{1}, a_{3}\right) \in G_{b_{1}, b_{2}, b_{3}}^{\prime}\right\} \subset A_{b_{1}} \times\left[\frac{b_{2}-b_{1}}{b_{3}-b_{2}} A_{b_{3}}\right]_{\delta} .
$$

Recall that $[r]_{\delta}$ stands for the largest number of the form $\delta n, n \in \mathbb{Z}$, with $\delta n \leq r$, and $[R]_{\delta}:=\left\{[r]_{\delta}: r \in R\right\}$. It follows easily from (4.51) (and recalling $A_{b_{1}} \subset \delta \mathbb{Z}$ ) that

$$
\left|\left\{t_{1}+t_{2}:\left(t_{1}, t_{2}\right) \in F_{b_{1}, b_{2}, b_{3}}\right\}\right| \lesssim \delta^{-s} \text {. }
$$

Moreover, since $\left|\left(b_{2}-b_{1}\right) /\left(b_{3}-b_{2}\right)\right| \approx 1$ for every triple $\left(b_{1}, b_{2}, b_{3}\right)$ satisfying (4.49), it follows that $\left|F_{b_{1}, b_{2}, b_{3}}\right| \approx \delta^{-2 s}$ whenever (4.49) holds and $\left|G_{b_{1}, b_{2}, b_{3}}^{\prime}\right| \approx \delta^{-2 s}$. For such a good 
triple $\left(b_{1}, b_{2}, b_{3}\right)$, the Balog-Szemerédi-Gowers theorem, Theorem 4.38, implies that there exist subsets

$$
D_{b_{1}, b_{2}, b_{3}}^{1} \subset A_{b_{1}} \quad \text { and } \quad \tilde{D}_{b_{1}, b_{2}, b_{3}}^{2} \subset\left[\frac{b_{2}-b_{1}}{b_{3}-b_{2}} A_{b_{3}}\right]_{\delta}
$$

such that $\left|D_{b_{1}, b_{2}, b_{3}}^{1}\right|,\left|\tilde{D}_{b_{1}, b_{2}, b_{3}}^{2}\right| \approx \delta^{-s}$,

$$
\left|\left(D_{b_{1}, b_{2}, b_{3}}^{1} \times \tilde{D}_{b_{1}, b_{2}, b_{3}}^{2}\right) \cap F_{b_{1}, b_{2}, b_{3}}\right| \approx \delta^{-2 s}
$$

and

$$
\left|D_{b_{1}, b_{2}, b_{3}}^{1}+\tilde{D}_{b_{1}, b_{2}, b_{3}}^{2}\right| \lesssim \delta^{-s}
$$

Let

$$
D_{b_{1}, b_{2}, b_{3}}^{2}:=\left\{a_{3} \in A_{b_{3}}:\left[\frac{b_{2}-b_{1}}{b_{3}-b_{2}} a_{3}\right]_{\delta} \in \tilde{D}_{b_{1}, b_{2}, b_{3}}^{2}\right\} .
$$

It then follows from the definition of $F_{b_{1}, b_{2}, b_{3}}$ and (4.52) that

$$
\left|G_{b_{1}, b_{2}, b_{3}}\right|:=\left|\left(D_{b_{1}, b_{2}, b_{3}}^{1} \times D_{b_{1}, b_{2}, b_{3}}^{2}\right) \cap G_{b_{1}, b_{2}, b_{3}}^{\prime}\right| \approx \delta^{-2 s} .
$$

for a good triple $\left(b_{1}, b_{2}, b_{3}\right)$. Moreover, (4.53) easily implies that

$$
N_{1}:=N\left(D_{b_{1}, b_{2}, b_{3}}^{1}+\frac{b_{2}-b_{1}}{b_{3}-b_{2}} D_{b_{1}, b_{2}, b_{3}}^{2}, \delta\right) \lesssim \delta^{-s} .
$$

Finally, combining (4.55) with the Plünnecke-Ruzsa inequality, Theorem 4.39, gives

$$
N_{2}:=N\left(D_{b_{1}, b_{2}, b_{3}}^{2}+D_{b_{1}, b_{2}, b_{3}}^{2}, \delta\right) \approx N\left(\frac{b_{2}-b_{1}}{b_{3}-b_{2}} D_{b_{1}, b_{2}, b_{3}}^{2}+\frac{b_{2}-b_{1}}{b_{3}-b_{2}} D_{b_{1}, b_{2}, b_{3}}^{2}, \delta\right) \lesssim \delta^{-s}
$$

for any good triple $\left(b_{1}, b_{2}, b_{3}\right)$. Since there are $\approx|B|^{3}$ good triples $\left(b_{1}, b_{2}, b_{3}\right)$, one can find $b_{1}, b_{3}$ such that (4.54)-(4.56) hold for $\approx|B|$ choices of $b_{2}$ (and so that $\left(b_{1}, b_{2}, b_{3}\right)$ remains a good triple). Fix such $b_{1}, b_{3} \in B$. Then, a simple Cauchy-Schwarz argument (similar to the one before (4.16)) shows that $\left|G_{b_{1}, b_{2}, b_{3}} \cap G_{b_{1}, b_{2}^{\prime}, b_{3}}\right| \approx \delta^{-2 s}$ for $\approx|B|^{2}$ pairs $\left(b_{2}, b_{2}^{\prime}\right)$, with both $\left(b_{1}, b_{2}, b_{3}\right)$ and $\left(b_{1}, b_{2}^{\prime}, b_{3}\right)$ being good triples. Now, one can finally fix $b_{2} \in B$ such that $\left(b_{1}, b_{2}, b_{3}\right)$ is a good triple, and

$$
\left|G_{b}\right|:=\left|G_{b_{1}, b_{2}, b_{3}} \cap G_{b_{1}, b, b_{3}}\right| \approx \delta^{-2 s}
$$

for $\approx|B|$ choices of $b \in B$ such that $\left(b_{1}, b, b_{3}\right)$ is a good triple. I denote the set of $b \in B$ satisfying these conditions by $B_{0}$. With (4.55) in mind, write

$$
c_{b}:=\frac{b-b_{1}}{b_{3}-b}, \quad b \in B_{0},
$$

and abbreviate $c:=c_{b_{1}}$ (note that $|c|,\left|c_{b}\right| \approx 1$ for all $b \in B_{0}$ by (4.49)). Also, write

$$
D^{1}:=D_{b_{1}, b_{2}, b_{3}}^{1}(\delta) \text { and } D^{2}:=D_{b_{1}, b_{2}, b_{3}}^{2}(\delta),
$$

where $R(\delta)$ stands for the $\delta$-neighbourhood of $R \subset \mathbb{R}^{d}$. To complete the proof, I repeat an argument of Bourgain (see p. 219 in [2]). Assume for a moment that $x \in c D^{2} \times D^{2} \subset \mathbb{R}^{2}$ and $b \in B_{0}$. Then $\chi_{-G_{b}(\delta)-y}(x)=1$, whenever

$$
y \in-G_{b}(\delta)-x \subset-\left(D^{1} \times D^{2}\right)-\left(c D^{2} \times D^{2}\right)=-\left(D^{1}+c D^{2}\right) \times-\left(D^{2}+D^{2}\right),
$$


(the first inclusion uses (4.54) and (4.57)) and the Lebesgue measure of such choices $y$ is evidently $\mathcal{L}^{2}\left(G_{b}(\delta)\right)$. This gives the inequality

$$
\chi_{c D^{2}+c_{b} D^{2}} \leq \frac{1}{\mathcal{L}^{2}\left(G_{b}(\delta)\right)} \int_{-\left(D^{1}+c D^{2}\right) \times-\left(D^{2}+D^{2}\right)} \chi_{\pi_{b_{1}, b, b_{3}}\left(-G_{b}(\delta)\right)-\pi_{b_{1}, b, b_{3}}(y)} d y, \quad b \in B_{0},
$$

by the definition of $\pi_{b_{1}, b, b_{3}}$ (see (4.50)). Indeed, if $t \in c D^{2}+c_{b} D^{2}=\pi_{b_{1}, b, b_{3}}\left(c D^{2} \times D^{2}\right)$, then $t=\pi_{b_{1}, b, b_{3}}(x)$ for some $x \in c D^{2} \times D^{2}$. As discussed above,

$$
t=\pi_{b_{1}, b, b_{3}}(x) \in \pi_{b_{1}, b, b_{3}}\left(-G_{b}(\delta)-y\right)=\pi_{b_{1}, b, b_{3}}\left(-G_{b}(\delta)\right)-\pi_{b_{1}, b, b_{3}}(y)
$$

whenever for $y \in-G_{b}(\delta)-x \subset-\left(D^{1}+c D^{2}\right) \times-\left(D^{2}+D^{2}\right)$, and the set of such points $y$ has measure $\mathcal{L}^{2}\left(G_{b}(\delta)\right)$.

Finally, integrating inequality (4.58) and recalling (4.55), (4.56), (4.51) and (4.57), one obtains

$$
\mathcal{L}^{1}\left(c D^{2}+c_{b} D^{2}\right) \lesssim \frac{\left(N_{1} \delta\right)\left(N_{2} \delta\right)}{\mathcal{L}^{2}\left(G_{b}(\delta)\right)} \mathcal{L}^{1}\left(\pi_{b_{1}, b, b_{2}}\left(G_{b}\right)\right) \lesssim \delta^{1-s}, \quad b \in B_{0} .
$$

However, $D^{2} \times D^{2}$ is the $\delta$-neighbourhood of a generalised $(\delta, 2 s)$-set in the plane, so Bourgain's discretized projection theorem, Theorem 5 in [2], can be applied with $\alpha:=$ $2 s<2=: d$. If $\mu_{1}$ is the normalised counting measure on the set $\left\{c_{b} / c: b \in B_{0}\right\}$, then it is not hard to check that $\mu_{1}$ satisfies assumption (0.14) from [2] for any $\tau_{0}>0$ and some $\kappa>$ 0 depending only on $\tau$ (using the fact that $B_{0}$ is a $(\delta, \tau)$-set with $\left|B_{0}\right| \approx \delta^{-\tau}$; if preferred, this is even easier to check, if one first reduces $B_{0}$ slightly so that the the derivative of $b \mapsto c_{b}$ has absolute value $\approx 1$ uniformly for $b \in B_{0}$ ). Thus, the conclusion (0.19) of [2] states that some $c_{b} / c$ with $b \in B_{0}$ should satisfy $\mathcal{L}^{1}\left(D^{2}+\left(c_{b} / c\right) D^{2}\right) \geq \delta^{1-s-\epsilon_{2}}$ for some constant $\epsilon_{2}>0$ depending only on $s$ and $\tau$. Recalling that $|c| \approx 1$, this evidently violates (4.59). A contradiction is reached, and the proof of Proposition 4.36 is complete.

\section{REFERENCES}

[1] J. Bourgain: On the Erdős-Volkmann and Katz-Tao ring conjectures, Geom. Funct. Anal. 13 (2003), 334-365

[2] J. BourgAin: The discretised sum-product and projection theorems, J. Anal. Math 112 (2010), pp. 193-236

[3] J. ElLENBERG AND D. ERMAN: Furstenberg sets and Furstenberg schemes over finite fields, to appear in Algebra Number Theory, available at arXiv:1502.03736

[4] G. A. Edgar And C. Miller: Borel Subrings of the Reals, Proc. Amer. Math. Soc. 131 (4) (2003), $1121-1129$

[5] K. Falconer, J. Fraser And Z. Jin: Sixty Years of Fractal Projections, in Fractal Geometry and Stochastics V, Vol. 70 of Progress in Probability, 3-25

[6] K. FAlCONER AND P. MATtila: Strong Marstrand theorems and dimensions of sets formed by subsets of hyperplanes, to appear in J. Fractal Geom., available at arXiv:1503.01284

[7] K. FÄSSLER AND T. ORPONEN: On restricted families of projections in $\mathbb{R}^{3}$, Proc. London Math. Soc. 109 (2) (2014), 353-381

[8] H. FurstenberG: Intersections of Cantor sets and transversality of semigroups, Problems in Analysis (Sympos. Salomon Bochner, Princeton Univ., Princeton, N.J., 1969), Princeton Univ. Press, Princeton, N.J., 1970, 41-59

[9] N. KATZ AND T. TAO: Some connections between Falconer's distance set conjecture, and sets of Furstenberg type, New York J. Math. 7 (2001), pp. 149-187

[10] N. KATZ, I. ŁABA AND T. TAO: An improved bound on the Minkowski dimension of Besicovitch sets in $\mathbb{R}^{3}$, Ann. of Math. 152 (2000), 383-446

[11] N. KATZ AND J. ZAHL: An improved bound on the Hausdorff dimension of Besicovitch sets in $\mathbb{R}^{3}$, preprint (2017), arXiv:1704.07210 
[12] R. Kaufman: On Hausdorff dimension of projections, Mathematika 15 (1968), 153-155

[13] R. Kaufman AND P. Mattila: Hausdorff dimension and exceptional sets of linear transformations, Ann. Acad. Sci. Fenn. Ser. A I Math. 1 (1975), 387-392

[14] J. M. MARSTRAnd: Some fundamental geometrical properties of plane sets of fractional dimensions, Proc. London Math. Soc.(3), 4, (1954), 257-302

[15] D. OBERLIN: Restricted Radon transforms and projections of planar sets, published electronically in Canadian Math. Bull. (2014), available at arXiv:0805.1678

[16] D. OberLin: Some toy Furstenberg sets and projections of the four-corner Cantor set, Proc. Amer. Math. Soc. 142 (4) (2013), 1209-2015

[17] T. Orponen: On the Packing Dimension and Category of Exceptional Sets of Orthogonal Projections, Ann. Mat. Pura Appl. 194 (3) (2015), 843-880

[18] T. ORPONEN: Projections of planar sets in well-separated directions, Adv. Math. 144 (8) (2016), 3419-3430

[19] T. ORPONEN: Improving Kaufman's exceptional set estimate for packing dimension, preprint (2016), available at arXiv:1610.06745

[20] P. SHMERKIN: On Furstenberg's intersection conjecture, self-similar measures, and the $L^{q}$ norms of convolutions, preprint (2016), arXiv:1609.07802v1

[21] T. TAO AND V. VU: Additive combinatorics, Cambridge University Press (2006)

[22] T. WolfF: Recent work connected with the Kakeya problem, in: Prospects in mathematics (Princeton, NJ, 1996), Amer. Math. Soc., Providence, RI, (1999), 129-162

[23] M. WU: A proof of Furstenberg's conjecture on the intersections of $\times p$ and $\times q$-invariant sets, preprint (2016), arXiv:1609.08053

[24] R. ZHANG: Polynomials with dense zero sets and discrete models of the Kakeya conjecture and the Furstenberg set problem, appeared online in Sel. Math. New Ser. (2016), available at arXiv:1403.1352

[25] R. ZHANG: On configurations where the Loomis-Whitney inequality is nearly sharp and applications to the Furstenberg set problem, Mathematika 61 (1) (2015), 145-161

UniVERSity OF HELSINKI, DePARTMENT OF MATHEMATICS AND STATISTICS

E-mail address: tuomas.orponen@helsinki.fi 